\title{
Energy-Efficient Multicast Transmission for Underlay Device-to-Device Communications: A Social-Aware Perspective
}

\author{
Fan Jiang, ${ }^{1,2}$ Yao Liu, ${ }^{2}$ Chenbi Li, ${ }^{1}$ and Changyin Sun ${ }^{1}$ \\ ${ }^{1}$ School of Communication and Information Engineering, Xi'an University of Posts and Telecommunications, Xian, China \\ ${ }^{2}$ Department of Computer Science and Engineering, University of South Florida, Tampa, FL, USA \\ Correspondence should be addressed to Fan Jiang; fjiangwbc@gmail.com
}

Received 11 December 2016; Accepted 19 February 2017; Published 27 March 2017

Academic Editor: Nan Zhao

Copyright (c) 2017 Fan Jiang et al. This is an open access article distributed under the Creative Commons Attribution License, which permits unrestricted use, distribution, and reproduction in any medium, provided the original work is properly cited.

\begin{abstract}
In this paper, by utilizing the social relationships among mobile users, we present a framework of energy-efficient cluster formation and resource allocation for multicast D2D transmission. In particular, we first deal with D2D multicast cluster/group formation strategy from both physical distance and social trust level. Then we aim to maximize the overall energy-efficiency of D2D multicast groups through resource allocation and power control scheme, which considers the quality-of-service (QoS) requirements of both cellular user equipment and D2D groups. A heuristic algorithm is proposed to solve above energy-efficiency problem with less complexity. After that, considering the limited battery capacity of mobile users, we propose an energy and social aware cluster head update algorithm, which incorporates both the energy constraint and social centrality measurement. Numerical results indicate that the proposed social-tie based D2D multicast group formation and update algorithm form a multicast group in an energy efficient way. Moreover, the proposed resource and power allocation scheme achieves better energy efficiency in terms of throughput per energy consumption. These results show that, by exploiting social domain information, underlay D2D multicast transmission has high practical potential in saving the source on wireless links and in the backhaul.
\end{abstract}

\section{Introduction}

With the vast proliferation of wireless smart devices, mobile traffic demand grows at an unprecedented speed than ever. According to Cisco statistics [1], there are more than 15 billion devices today on what is popularly referred to as the Internet of Things (IoT), and this number is expected to triple by the year 2020. In particular, the local area service of popular content sharing (e.g., video, music, and gaming) is one of the main reasons for this tremendous growth. The continuously increasing demand for wireless access especially data services for geographically proximate user has brought great challenges to current mobile communication infrastructure. Under this circumstance, the direct connectivity between mobile devices, namely, Device-to-Device (D2D) communication, emerges as a key component for the fifthgeneration $(5 G)$ wireless communication system $[2,3]$. In D2D communication, user equipment (UE) in the proximity region transmits data signals to each other over a direct link instead of through the base station (BS), which improves spectral utilization and saves power consumption $[4,5]$.

As one of the main features, D2D multicast transmission provides an effective solution to mitigate the burden of BS and improves transmission efficiency, which is important for scenarios such as content sharing, device discovery, and public safety $[6,7]$. D2D multicast communication underlaying cellular networks refers to the transmission scenario that a transmitter, namely, cluster head $(\mathrm{CH})$, sends the same packet to a group of destination UE items over the D2D links which reuse the resource of cellular links [8]. By exploiting the inherent broadcast nature of wireless channels, D2D multicast transmission disseminates the same content simultaneously to multiple recipients. Compared with traditional point-to-point unicast D2D communication, multicast D2D transmission reduces overhead and improves resource efficiency. However, multicast D2D transmission underlaying cellular network also has its own challenges. 
The first challenge faces with D2D multicast transmission are how to energy-efficiently form a specific multicast group and how to select an appropriate $\mathrm{CH}$ for each multicast group. Due to the increasing power consumption of information and communication technology (ICT) industry, the progress in battery technology of user terminals is rather slow. Given the limited battery capacity of mobile devices, energy-efficient solutions are imperative to be integrated into the D2D communications $[9,10]$. On the other hand, recent studies $[11,12]$ show that the social behaviors of human beings who carry the handheld communication devices can be leveraged to improve the performance of D2D communications. Consequently, it is worth discussing whether the knowledge of social characteristics can benefit the quality of service (QoS) of D2D multicast communication. In fact, improving the QoS might simultaneously increase the energy efficiency of D2D multicast [13]. Therefore, examining clustering strategy and $\mathrm{CH}$ selection method under the social-aware premise can reveal ways of reducing energy consumption without hindering the offered QoS of D2D multicast transmission [14].

Another challenge is that when users within a multicast group receive the same packets, the data rates of different receivers are different because of variant link conditions between each receiver and the $\mathrm{CH}$. If the $\mathrm{CH}$ transmits at a higher rate than the maximum rate that a user can handle, the user cannot decode the transmitted data at all. Therefore, it is commonly assumed that the $\mathrm{CH}$ should transmit at the lowest rate of all users within a multicast group determined by the user with the worst channel condition $[15,16]$. Obviously, this conventional assumption results in inefficient use of spectrum resource and power resource. In a multicast group, the situation is especially undesirable when most of the UE items are in good channel condition and capable of high data transmission, while only a small fraction of the UE items are suffering from deep fading. Thus, how to allocate resource within a multicast group so as to guarantee reliable QoS requirement of different UE is very essential. Besides, when D2D communication reuses the spectrum resource of the regular cellular users, effective interference management strategy is indispensable so as to deal with complex resource reuse interference. Hence, how to energy-efficiently allocate spectrum resource and power for D2D multicast groups is also a big challenge.

Among current contributions related to D2D multicast transmission, most of the works focus on how to mitigate the reuse interference through resource allocation and power control schemes [17-20]. Meshgi et al. [17] studied a joint channel and power allocation strategy which aims at maximizing the sum throughput of cellular users and D2D groups in a cell. Wu et al. [18] adopted a distance based channel allocation and power allocation scheme harnessing cognitive radio to maximize system capacity for D2D underlay multicast communication. Peng et al. [19] researched the problem of resource allocation involving with D2D multicast groups underlaying OFDMA systems. The paper establishes a joint optimization function which achieves maximum sum throughput and also considers how to protect the QoS requirement of cellular users. Bhardwaj and Agnihotri [20] proposed a scheme to minimize the interference among D2D users and cellular users through a resource allocation scheme. Huang et al. [21] utilized D2D multicast over the Wi-Fi network to offload traffic from cellular network. However, these above studies mainly utilize the physical domain information to maximize the network transmission rate, where users are supposed to be altruistic and eager to collaborate with each other. On the other hand, the social-aware based unicast D2D communications have received huge attentions in recent studies. For example, Li et al. [22] proposed a social-aware D2D resource sharing scheme that exploits social network properties of community and centrality to aid resource allocation. Zhao et al. [23] dealt with the resource allocation problem for D2D communications from the perspective of a social group utility game. Wang et al. [24] adopted a socialcommunity-aware resource allocation scheme by building a two-step coalition game to allocate cellular channels to D2D pairs for D2D communication underlaying cellular networks. Zhang et al. [25] utilized the social network characteristics to offload traffic from cellular networks. However, these above works only focus on the unicast scenario where multicast transmission scenario is not considered. Moreover, an aspect often been neglected in existing social-aware approaches for $\mathrm{D} 2 \mathrm{D}$ network is the energy consumption of the participating devices.

To the best of our knowledge, only a limited number of works in literature exploit social metrics to improve the effectiveness of D2D multicast communications. In the related researches, Cao et al. [26] proposed a social-aware cooperative video multicast framework to stimulate the cooperation behavior of mobile users. Orsino et al. [27] studied the social-aware content disseminate scenario with different transmission mode (e.g., D2D, unicast, and multicast), which aims at minimizing the information diffusion time of a given content. Zhang et al. [28] discussed the cluster formation and resource allocation problem in D2D networks which utilizes different duplex scheme to coordinate the channel sharing between the cellular links and the D2D links. However, the above works mainly focus on the system spectrum efficiency while ignoring the energy efficiency of D2D multicast transmission. Moreover, most studies do not address the problem of D2D multicast group formation.

By incorporating social-aware circumstance into D2D multicast transmission, this paper utilizes the social characteristics to assist multicast group formation and selects the best $\mathrm{CH}$ for each D2D multicast group based on energy efficiency. In particular, we first deal with how to quantify the immaterial social relationship between users; then both the social and network metrics are used in D2D multicast group formation process. After the multicast group has been formed, an energy-efficient resource allocation and power control scheme is proposed for D2D multicasting scenario, which considers the QoS requirements of different users. Moreover, based on the social ties as well as remaining energy, a social and energy-aware cluster head update algorithm is proposed so as to reselect a suitable $\mathrm{CH}$ for each D2D multicast group. Extensive simulations demonstrate the effectiveness of our proposed scheme, which significantly improves the system performance compared with existing schemes. 

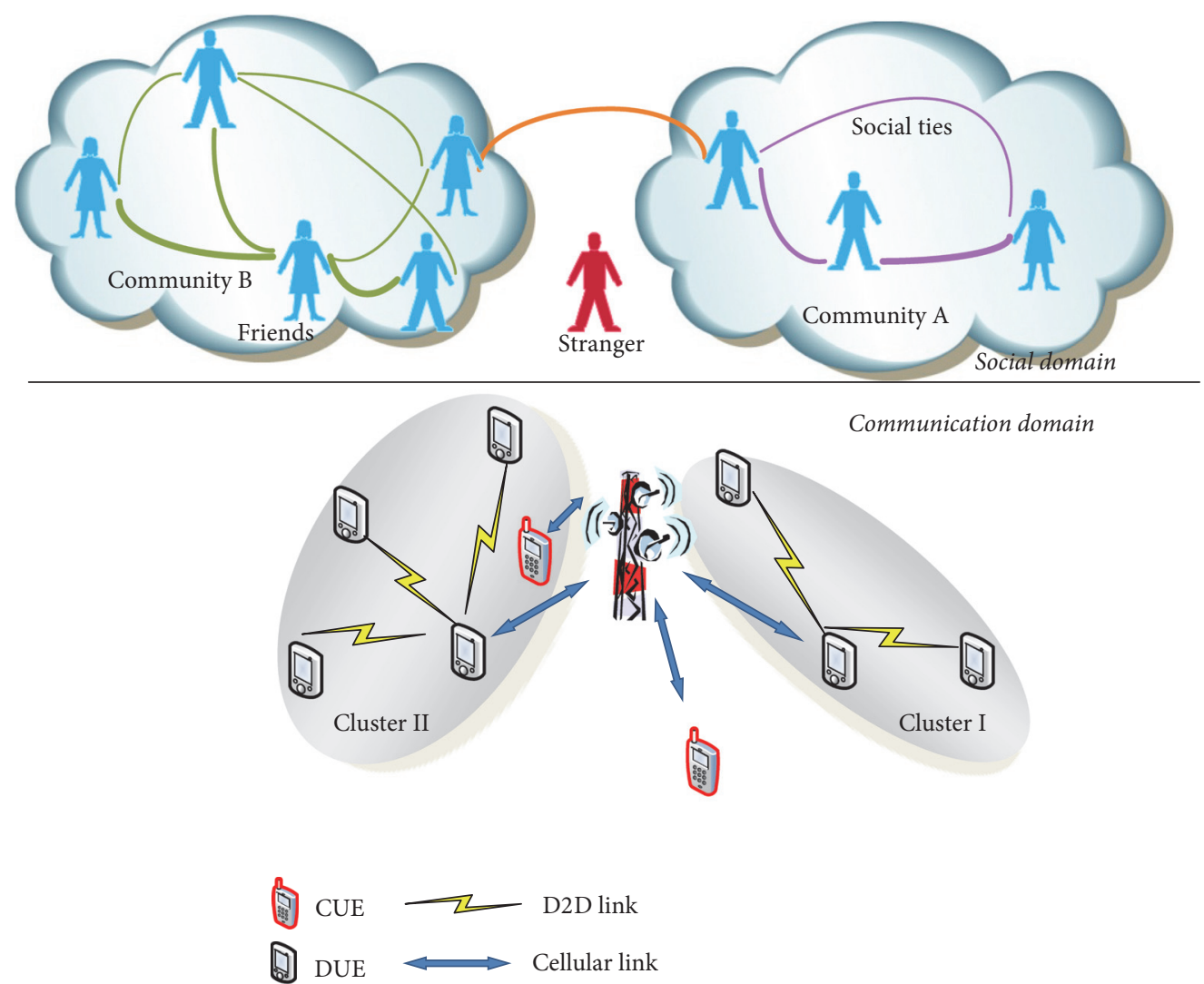

FIGURE 1: Social-aware D2D system projected onto two domains.

The remainder of the paper is organized as follows. In Section 2, we describe the social and network model and illustrate the multicast group formation algorithm from both physical and social domain information. Section 3 investigates the energy-efficient resource and power allocation problem and a heuristic algorithm is then proposed with less complexity. The proposed social-aware cluster head update algorithm is presented in Section 4. In Section 5, simulation results for the proposed scheme are presented. Finally, Section 6 concludes the paper.

\section{System Model and Social Tie Based D2D Multicast Group Formation}

In this section, we first introduce the social and network model and then propose a social tie based D2D multicast group formation strategy for D2D multicast transmission underlay a cellular network.

2.1. Social and Network Model. In real life, wireless terminals are carried and used by human beings who exhibit certain social interests by forming steady social networks. In a so-called social network, participants who have common interests or similar background are prone to build a reliable relationship through social interaction with each other. Naturally, those people who have the common social characteristic might form a real social community. In particular, regarding online social network services, such as Facebook, Twitter, and LinkedIn, mobile users might strengthen their social relationship through sharing the common interested contents (e.g., video, music, and photos) in the same community. Take a popular mobile application "Wechat" as an example. Friends in the same social community exhibit their preference in terms of content publishing. Those people who have the similar interests in the community might show their enthusiasm and endorsement through the behavior of content sharing and thumb up. Consequently, such potential social ties among different mobile users can be utilized to improve the effectiveness of D2D transmission. By introducing the socialaware information into D2D multicast communications, D2D users who have the similar interests might be prone to join the same community and obtain the similar contents through short range link from a D2D cluster head other than through BS. This helps to retrieve the BS from multicast load burden and greatly improves spectrum efficiency [29].

As shown in Figure 1, we consider a social-aware D2D communication underlaying cellular network model which combines virtual social domain and physical communication domain. In the social domain, people are divided into different communities according to their social relationships. It is obvious that members of the same community are prone to contact more frequently and are considered as trusted friends, where newly arrived strangers are often excluded from the 
communities. Moreover, there are also some people who serve as bridges between different communities. In particular, as illustrated in Figure 1, a set of thin and thick lines among people represent the strength of social ties between friends. These ties are long-term characteristics which can reveal their willingness to participate in cooperation (e.g., behavior such as sharing the common interested content or serving as a relay for others). The closer the relationship between two friends is, the more likely they will provide service for each other.

Each human being in the social domain is projected as a UE in the communication domain. For simplicity, when we mention a UE in the physical domain, we also refer to its corresponding human being in the social domain. In the physical domain, a BS is located at the center of the cellular cell which has no corresponding role in the social network. The BS is responsible for collecting channel information and allocating spectrum resource for each UE. UE is divided into two categories: a regular cellular user equipment (CUE) refers to the UE that communicates directly with the BS; each CUE is allocated with a unique channel, while D2D user equipment (DUE) refers to the UE items that exchange data to each other over the direct wireless link. Since UE items in the same community contact more frequently than those in other communities, we focus on the scenario that a DUE pair belongs to the same community. Furthermore, for the stranger in the social domain, it is assumed as a regular CUE in the physical domain since D2D communication is unavailable due to trust issues.

Combined with the information provided by both social domain and physical domain, we can incorporate the user's social relationship to facilitate the D2D multicasting process. More specifically, the formation of D2D multicast group can rely on both the social ties among different users in communities and the actual physical distance between DUE items. Furthermore, centrality metrics characterizing the importance of users in social networks can also be utilized for socialaware $\mathrm{CH}$ selection. However, before using such social-aware information, we need to first quantify such immaterial social ties and incorporate them into our physical model.

We consider a general scenario that a number of UE items in proximity want to share content with their peers (e.g., videos of Olympic Games). The shared content is either UE originated or obtained from the cellular network. For example, UE items transmit their own photos to their friends or share videos which are previously downloaded via the cellular connections. Under such scenario, a multicast D2D group can be organized. The UE that has stored the data can act as a source node to disseminate the content requirement to a multiple of familiar UE items through D2D communication.

Suppose all of the active DUE items constituting a set D, where $\mathrm{D}=\left\{d_{1}, d_{2}, \ldots, d_{M}\right\}$. Note that according to the considered scenario, CUE might also join the D2D multicast group during the $\mathrm{D} 2 \mathrm{D}$ multicast process. To improve the spectrum efficiency, we assume that D2D communication is supported in the underlay mode, which means DUE shares uplink resources with ongoing cellular communications. For any DUE $d_{i}, \forall d_{i} \in D$, suppose it has $K$ neighbor DUE within its maximum transmission range, which can be described as a neighbor set and denoted as $K^{d_{i}}=\left\{k_{1}^{d_{i}}, k_{2}^{d_{i}}, \ldots, k_{K}^{d_{i}}\right\}$. We exploit an undirected weighted graph $G_{d_{i}}=\left(V_{d_{i}}, \varepsilon_{d_{i}}\right)$ to model the physical-social relationship between DUE $d_{i}$ and its neighbor DUE $k_{j}^{d_{i}}, \forall k_{j}^{d_{i}} \in K^{d_{i}}$, where $V_{d_{i}}$ represents the vertex set and $\varepsilon_{d_{i}}$ denotes the edge set. In graph $G_{d_{i}}$, the vertex set is built as $V_{d_{i}}=\left\{d_{i}, k_{1}^{d_{i}}, k_{2}^{d_{i}}, \ldots, k_{K}^{d_{i}}\right\}$, and the set of edges $\varepsilon_{d_{i}}$ is represented as $\varepsilon_{d_{i}}=\left\{\left(d_{i}, k_{j}^{d_{i}}\right): e_{d_{i}, k_{j}^{d_{i}}}=1, \forall d_{i} \in \mathrm{D}\right.$, $\left.\forall k_{j}^{d_{i}} \in K^{d_{i}}\right\}$, where $e_{d_{i}, k_{j}^{d_{i}}}=1$ if and only if $k_{j}^{d_{i}}$ lies within the transmission range of $d_{i}$.

After completion of the graph $G_{d_{i}}$, DUE items that are physically in proximity have been decided. We then deal with the weighted coefficient between each DUE pair from the aspect of social characteristic. We define the social tie strength as the weight of each edge in $\varepsilon_{d_{i}}$. In particular, a weight coefficient $\omega_{d_{i}, k_{j}^{d_{i}}}$ is introduced to denote the strength of social relationship between $d_{i}$ and $k_{j}^{d_{i}}$, where $\omega_{d_{i}, k_{j}^{d_{i}}} \in$ $[0,1]$. A larger value of $\omega_{d_{i}}, k_{j}^{d_{i}}$ represents a stronger social relationship between two DUE items, while on the other hand, a smaller value of $\omega_{d_{i}, k_{j}^{d_{i}}}$ might represent a weaker social tie between two DUE items. Since D2D multicast transmission usually happens in close proximity regions where UE items are acquainted with each other and eager to share common interested content. It is reasonable to assume that the strength of social ties among users largely impacts UE's eagerness to participate in the multicast transmission besides the physical distance. Combined with the proposed system model, the problem becomes how to quantify the weight coefficient $\omega_{d_{i}, k_{j}^{d_{i}}}$ between each DUE pair.

Typically, factors that affect social relationships can be divided into two aspects: time factor and interactivity factor [29]. Time factor refers to the impact of time on the social relationships, while interactivity factor reflects the quality of the interpersonal communication, such as contents to be disseminated and the way of communication. Here, we adopt the average contact duration $\overline{D_{d_{i}, k_{j}^{d_{i}}}}$ to describe the time factor, which is represented as

$$
\overline{D_{d_{i}, k_{j}^{d_{i}}}}=\frac{\int_{0}^{\varphi} \delta_{d_{i}, k_{j}^{d_{i}}}(t) d t}{R_{d_{i}, k_{j}^{d_{i}}}} .
$$

$\overline{D_{d_{i}, k_{j}^{d_{i}}}}$ reflects the time and frequency of contact between two DUE items comprehensively, where $\varphi$ denotes the observation time period and $R_{d_{i}, k_{j}^{d_{i}}}$ is the number of contact times between $d_{i}$ and its neighbor user $k_{j}^{d_{i}}$ during time period $\varphi$. $\delta_{d_{i}, k_{j}^{d_{i}}}(t)=1$ if $d_{i}$ and $k_{j}^{d_{i}}$ communicate within the observation period; otherwise, $\delta_{d_{i}, k_{j}^{d_{i}}}(t)=0$. By normalizing (1) [30], we can obtain the time factor $C_{d_{i}, k_{j}^{d_{i}}}$ as

$$
C_{d_{i}, k_{j}^{d_{i}}}=\exp \left(-\frac{\left(\overline{D_{d_{i}, k_{j}^{d_{i}}}}\right)^{2}}{2 \delta^{2}}\right),
$$

where $\delta$ denotes scale parameter of contact duration. 
On the other hand, interactivity factor relies on the quality and familiarity of the interpersonal interaction. In particular, it is well founded that if $d_{i}$ and $k_{j}^{d_{i}}$ have more common friends, they are more likely to have a closer social relationship. Therefore, we use common friend's index $F_{d_{i}, k_{j}^{d_{i}}}$ [31] to represent the interactivity factor:

$$
F_{d_{i}, k_{j}^{d_{i}}}=\frac{\left|\mathscr{F}_{d_{i}} \cap \mathscr{F}_{k_{j}^{d_{i}}}\right|}{\left|\mathscr{F}_{d_{i}} \cup \mathscr{F}_{k_{j}^{d_{i}}}\right|},
$$

where $|\bullet|$ is the cardinality of the set and $\mathscr{F}_{d_{i}}$ and $\mathscr{F}_{k_{j}^{d_{i}}}$ stand for the friends set of $d_{i}$ and $k_{j}^{d_{i}}$, respectively. It is obvious that $F_{d_{i}, k_{j}^{d_{i}}}$ reveals the degree of intimacy between two UE items, which helps $d_{i}$ to decide whether to participate in multicast transmission.

Accordingly, combined with time factor and interactivity factor, the weight coefficient $\omega_{d_{i}, k_{j}^{d_{i}}}$ which represents the social tie strength is expressed as

$$
\omega_{d_{i}, k_{j}^{d_{i}}}=\beta_{d_{i}} C_{d_{i}, k_{j}^{d_{i}}}+\left(1-\beta_{d_{i}}\right) F_{d_{i}, k_{j}^{d_{i}}},
$$

where $\beta_{d_{i}}, \beta_{d_{i}} \in[0,1]$ is a relative weight to balance the impact between $C_{d_{i}, k_{j}^{d_{i}}}$ and $F_{d_{i}, k_{j}^{d_{i}}}$. Here, $d_{i}$ is introduced to adjust the value of $\beta_{d_{i}}$ based on the real applications. Moreover, each of $d_{i}$ and $k_{j}^{d_{i}}$ independently stores and periodically updates these factors, that is, $\overline{D_{d_{i}, k_{j}^{d_{i}}}}, \mathscr{F}_{d_{i}}$, and $\mathscr{F}_{k_{j}^{d_{i}}}$, respectively.

2.2. Social Tie Based D2D Multicast Group Formation. The above physical and social domain information provides guidance for the multicast group formation issues in more open environment. More specifically, if a DUE wants to form a multicast group and disseminates the same content to a number of DUE items through D2D communication, the physical and social relationship between DUE items should be leveraged. Suppose an arbitrary DUE $d_{i}, \forall d_{i} \in \mathrm{D}$ wants to form a multicast group so as to share the common interested contents. DUE $d_{i}$ should check both the physical network availability and social trust level of its peers. This means, with the help of graph $G_{d_{i}}$, DUE $d_{i}$ can obtain both the physical and social information about its $K$ neighbor DUE. According to previous analysis, the set of edges in $\varepsilon_{d_{i}}$ reflects the physical distance between two DUE items where only those DUE items that locate at the transmission range of DUE $d_{i}$ can join in the multicast group in the physical domain. From the social domain, the weight of each edge in $\varepsilon_{d_{i}}$ represents the social tie strength between two DUE items. Based on above information, we put forward a social tie based multicast group formation strategy; the detailed process is described as follows.

Step 1. A DUE $d_{i}$ checks graph $G_{d_{i}}$ and removes the unavailable DUE in physical domain if $e_{d_{i}, k_{j}^{d_{i}}}=0$, where $k_{j}^{d_{i}}$ stands for neighbor DUE.
Step 2. DUE $d_{i}$ checks the weight coefficient $\omega_{d_{i}, k_{j}^{d_{i}}}$ in the DUE pair and removes the DUE from the remaining DUE sets, if $\omega_{d_{i}, k_{j}^{d_{i}}}<\Gamma_{d_{i}}^{\text {So }}$.

Here, $\Gamma_{d_{i}}^{\text {So }}$ represents a social threshold for each DUE, which is expressed as

$$
\Gamma_{d_{i}}^{\text {So }}=\frac{\rho \sum_{i=1}^{L} \omega_{d_{i}, k_{j}^{d_{i}}}}{L} .
$$

In expression (5), $\rho$ is the percentage value which is used to adjust social threshold, where $\rho \in[0,1]$. $L$ represents the total number of remaining DUE after Step 1 , where $L \leq K$. It can be derived that $\Gamma_{d_{i}}^{\text {So }}$ stands for the arithmetic mean of weight coefficient between DUE and its neighbor DUE. Accordingly, for those DUE items that might be physically close to DUE $d_{i}$ but not socially familiar with DUE $d_{i}$, multicast transmission is excluded. This is because if the social tie between two DUE items is weak, there might be trust issues where UE items are reluctant to cooperate by giving access of their devices to unfamiliar UE or following the circulation of their own data via other devices [32].

After above two steps, a multicast group is formulated for DUE $d_{i}$. Specifically, DUE items that are physically in proximity and have strong social connections with DUE $d_{i}$ will join in the same multicast group, where unavailable DUE items are removed. As a result, after the multicast group formation, DUE $d_{i}$ that originates the D2D multicast group serves as the $\mathrm{CH}$ and prepares for upcoming D2D multicast transmission. Naturally, the next step involves resource and power allocation for D2D multicast transmission. However, related to current social-aware approaches for D2D multicast transmission, the energy consumption of participating devices, especially the energy condition of the $\mathrm{CH}$, is always being neglected. Given the limited battery capacity of mobile devices, there exists the contingency that the existing spectrum and power allocation are not acceptable in D2D multicast scenarios due to high energy consumption [17]. Therefore, resource and power allocation strategy for D2D multicast transmission need to be energy-ware, at the same time considering user's social information, so as to provide an energy-efficient D2D resource management scheme $[33,34]$. In next section, we will develop an energy-aware resource and power allocation scheme for D2D multicast transmission.

\section{Resource and Power Allocation Scheme for Multicast D2D Transmission with Energy Efficiency Improvement}

After social-aware based multicast group has been accomplished, we consider the energy-aware resource and power allocation scheme for D2D multicast transmission underlaying uplink cellular network. Orthogonal frequency division multiple access (OFDMA) technique is introduced so that there is no intracell interference between the CUE items within the same cell. Moreover, BS is supposed to have the perfect channel state information (CSI) of all links. Assume 
$M$ groups of multicast DUE items coexist with $N$ CUE, where $M \leq N$. We consider a fully loaded cellular network scenario. This means there are $N$ channels and each CUE can only occupy one orthogonal channel. We use $n \in$ $\mathcal{N}=\{1,2, \ldots, N\}$ to indicate the $n$th CUE and also the channel it occupies, where $\mathcal{N}$ represents the set of CUE items. Parameter $m, m \in \mathscr{M}=\{1,2, \ldots, M\}$ is used to index $m$ th D2D group, where $\mathscr{M}$ is the set of DUE multicast groups after D2D multicast group formation. In each D2D multicast group, $\mathrm{CH}$ transmits message to a group of receivers within the same multicast group, and each receiver belongs to only one multicast group. We use $\mathscr{D}_{m}$ to represent the set of D2D receivers in the $m$ th D2D multicast group, where $\left|\mathscr{D}_{m}\right|$ is the total number of receivers in the $m$ th group. When $\left|\mathscr{D}_{m}\right|=1$, the scenario becomes unicast.

We define a set of binary variables $\rho_{m, n}$. If the $m$ th D2D group reuses the cellular channel of $n$th CUE, then $\rho_{m, n}=1$; otherwise, $\rho_{m, n}=0$. Assume that one D2D multicast group reuses at most one cellular channel, and each cellular channel can be reused at most by one D2D multicast group. Then we have

$$
\begin{aligned}
& \sum_{n=1}^{N} \rho_{m, n} \leq 1, \quad \forall m \in \mathscr{M}, \\
& \sum_{m=1}^{M} \rho_{m, n} \leq 1, \quad \forall n \in \mathcal{N} .
\end{aligned}
$$

Consequently, the channel quality of the $k$ th DUE as a receiver in $m$ th D2D group that reuse the $n$th cellular channel is given by

$$
\begin{aligned}
\gamma_{i, k}^{\mathrm{D} 2 \mathrm{D}_{m}}=\frac{G_{i, k}^{\mathrm{D} 2 \mathrm{D}}}{\sum_{n=1}^{N} \rho_{m, n} P_{n}^{\mathrm{CUE}} G_{n, k}^{\mathrm{C} 2 \mathrm{D}}+\sigma_{n}^{2}} & \\
& \forall i \in m, k \in \mathscr{D}_{m}, n \in \mathcal{N},
\end{aligned}
$$

where $\gamma_{i, k}^{\mathrm{D}^{2} \mathrm{D}_{m}}$ denotes the channel quality of $k$ th DUE as a multicast receiver in $m$ th D2D group when DUE $i$ serves as a $\mathrm{CH} . P_{n}^{\mathrm{CUE}}$ represents the transmit power of cochannel CUE $n$ that shares uplink cellular resource together. $G_{i, k}^{\mathrm{D} 2 \mathrm{D}}$ and $G_{n, k}^{\mathrm{C} 2 \mathrm{D}}$ stand for the channel gain between $\mathrm{CH} i$ and DUE $k$ in the $m$ th D2D group, between interfering CUE $n$ to DUE $k$, respectively. $\sigma_{n}^{2}$ denotes the noise power.

Similarly, for a CUE $n$, the channel quality is given by

$$
\gamma_{n}^{\mathrm{CUE}}=\frac{G_{n, \mathrm{BS}}^{\mathrm{CUE}}}{\sum_{m=1}^{M} \rho_{m, n} P_{i}^{\mathrm{D} 2 \mathrm{D}_{m}} G_{i, \mathrm{BS}}^{\mathrm{D} 2 \mathrm{C}}+\sigma_{n}^{2}}, \quad \forall i \in m, n \in \mathcal{N}
$$

here, $P_{i}^{\mathrm{D}^{2} \mathrm{D}_{m}}$ stands for the transmit power of interfering DUE $i$ that shares channel $n$ in $m$ th $\mathrm{D} 2 \mathrm{D}$ group. $G_{n, \mathrm{BS}}^{\mathrm{CUE}}$ and $G_{i, \mathrm{BS}}^{\mathrm{D} 2 \mathrm{C}}$ represent the link gain of CUE $n$ to the BS and cochannel DUE $i$ to the BS, respectively.

For the multicast transmission, the transmission rate is actually determined by the receiver with the worst channel condition. More specifically, for the $m$ th D2D group, the transmission condition at channel $n$ is given by

$$
\gamma_{m, n}^{\mathrm{D} 2 \mathrm{D}}=\min _{\forall i \in m, k \in \mathscr{D}_{m}} \gamma_{i, k}^{{\mathrm{D} 2 \mathrm{D}_{m}}}
$$

Therefore, the normalized transmission rate of the $m$ th D2D group is given by

$$
r_{m}^{\mathrm{D} 2 \mathrm{D}}=\sum_{n=1}^{N} \rho_{m, n} \log _{2}\left(1+P_{i}^{\mathrm{D} 2 \mathrm{D}_{m}} \gamma_{m, n}^{\mathrm{D} 2 \mathrm{D}}\right)
$$

The aggregate transmission rate of $m$ th $\mathrm{D} 2 \mathrm{D}$ group is then expressed as

$$
R_{m}^{\mathrm{D} 2 \mathrm{D}}=\left|\mathscr{D}_{m}\right| r_{m}^{\mathrm{D} 2 \mathrm{D}}
$$

Similarly, the normalized transmission rate for a CUE $n$ is expressed as

$$
R_{n}^{\mathrm{CUE}}=\log _{2}\left(1+P_{n}^{\mathrm{CUE}} \gamma_{n}^{\mathrm{CUE}}\right) .
$$

To guarantee reliable transmission of each UE, a threshold is set for the minimum signal-to-interference-and-noise ratio (SINR) requirement. Specifically, for the $m$ th D2D group, we can express the above requirement as

$$
P_{i}^{\mathrm{D} 2 \mathrm{D}_{m}} \gamma_{m, n}^{\mathrm{D} 2 \mathrm{D}} \geq \xi_{\min }^{\mathrm{D} 2 \mathrm{D}}
$$

For a CUE, the requirement is set as

$$
P_{n}^{\mathrm{CUE}} \gamma_{n}^{\mathrm{CUE}} \geq \xi_{\mathrm{min}}^{\mathrm{CUE}}
$$

where $\xi_{\min }^{\mathrm{D} 2 \mathrm{D}}$ and $\xi_{\min }^{\mathrm{CUE}}$ represent the minimum SINR threshold for reliable D2D multicast and CUE transmission specified by the system.

Moreover, combined with the maximum power constraints for CUE and D2D groups, and by substituting expressions (7) and (8) into (13) and (14), we can obtain the power transmission range of a $\mathrm{CH}$ and a $\mathrm{CUE}$ as

$$
\begin{aligned}
& \frac{\xi_{\min }^{\mathrm{D} 2 \mathrm{D}}}{\min \left(G_{i, k}^{\mathrm{D} 2 \mathrm{D}} /\left(\sum_{n=1}^{N} \rho_{m, n} P_{n}^{\mathrm{CUE}} G_{n, k}^{\mathrm{C} 2 \mathrm{D}}+\sigma_{n}^{2}\right)\right)} \leq P_{i}^{\mathrm{D} 2 \mathrm{D}_{m}} \\
& \leq P_{\max }^{\mathrm{DUE}}, \quad \forall i \in m, k \in \mathscr{D}_{m}, \\
& \frac{\xi_{\min }^{\mathrm{CUE}}}{G_{n, \mathrm{BS}}^{\mathrm{CUE}} /\left(\sum_{m=1}^{M} \rho_{m, n} P_{i}^{\mathrm{D} 2 \mathrm{D}_{m}} G_{i, \mathrm{BS}}^{\mathrm{D} 2 \mathrm{C}}+\sigma_{n}^{2}\right)} \leq P_{n}^{\mathrm{CUE}} \leq P_{\max }^{\mathrm{CUE}},
\end{aligned}
$$

$$
\forall i \in m, n \in \mathcal{N}
$$

where $P_{\max }^{\mathrm{CUE}}$ and $P_{\max }^{\mathrm{DUE}}$ denote the maximum allowed transmit power of CUE and DUE, respectively. From expression (15), we can infer that, besides the channel condition factor, the transmit power of a $\mathrm{CH}$ and the transmit power of a CUE that shares resource with a D2D multicast group are interacting with each other. In order to guarantee reliable communication, the transmit power of different kinds of UE should be controlled. 
Considering the limited energy capacity of each devices, our object is to maximize the energy efficiency (EE) of D2D multicast communication through appropriate resource and power allocation method. Combing (6)-(15), we formulate the resource and power allocation problem to maximize the overall energy efficiency of D2D groups as

$$
\begin{array}{ll}
\max _{\forall m \in \mathscr{M}} & \left(\frac{\sum_{m=1}^{M} R_{m}^{\mathrm{D} 2 \mathrm{D}}}{\sum_{m=1}^{M} P_{m}^{\mathrm{D} 2 \mathrm{D}_{m}}}\right) \\
\text { s.t. } & R_{m}^{\mathrm{D} 2 \mathrm{D}}=\left|\mathscr{D}_{m}\right| \sum_{n=1}^{N} \rho_{m, n} \log _{2}\left(1+P_{m}^{\mathrm{D} 2 \mathrm{D}_{m}} \gamma_{m, n}^{\mathrm{D} 2 \mathrm{D}}\right), \quad \forall m \in \mathscr{M}, n \in \mathscr{N} \\
& P_{m}^{\mathrm{D} 2 \mathrm{D}_{m}} \gamma_{m, n}^{\mathrm{D} 2 \mathrm{D}} \geq \xi_{\min }^{\mathrm{D} 2 \mathrm{D}} \\
& \gamma_{m, n}^{\mathrm{D} 2 \mathrm{D}}=\frac{G_{i, k}^{\mathrm{D} 2 \mathrm{D}}}{\sum_{n=1}^{N} \rho_{m, n} P_{n}^{\mathrm{CUE}} G_{n, m}^{\mathrm{C} 2 \mathrm{D}}+\sigma_{n}^{2}}, \quad \forall i \in m, k \in \mathscr{D}_{m}, n \in \mathcal{N} \\
& \sum_{n=1}^{N} \rho_{m, n} \leq 1, \quad \forall m \in \mathscr{M}_{m} \\
& \frac{\xi_{\min }^{\mathrm{D} 2 \mathrm{D}}}{\min \left(G_{m, k}^{\mathrm{D} 2 \mathrm{D}} /\left(\sum_{n=1}^{N} \rho_{m, n} P_{n}^{\mathrm{CUE}} G_{n, k}^{\mathrm{C} 2 \mathrm{D}}+\sigma_{n}^{2}\right)\right)} \leq P_{m}^{\mathrm{D} 2 \mathrm{D}_{m}} \leq P_{\max }^{\mathrm{DUE}}, \quad \forall m \in \mathscr{M},
\end{array}
$$

where $P_{m}^{\mathrm{D} 2 \mathrm{D}_{m}}$ represents the transmit power of $\mathrm{CH}$ that belongs to $m$ th D2D multicast group. Regarding [35], the object function in (16) gives the overall energy efficiency of all D2D multicast groups, where the energy efficiency is expressed as the sum of the transmission rate for all D2D multicast groups to the sum of total transmit power. Consequently, the overall energy efficiency is directly proportional to the aggregate transmission rate and inversely proportional to the transmit power. In order to improve the total energy efficiency, D2D multicast groups have to either decrease transmit power or increase the aggregate transmission rate. However, these two requirements are actually intertwined with each other. This is because from (11) we can infer that to decrease transmit power also contributes to decreased aggregate transmission rate. On the other hand, the increased aggregate transmission rate is also closely related to increased transmit power. Furthermore, for the latter requirement, it is actually determined by joint resource allocation and power allocation method. This is because when D2D multicast group reuses the uplink resources of cellular users, the resource reuse relationships between CUE and D2D multicast groups as well as transmit power of different UE directly impact the total throughput distribution of D2D multicast groups.

As a matter of fact, when D2D multicast transmission is undelaying with a cellular network, the resource allocation and power allocation strategy are actually coupled with each other. Once the resource pairing relationship between CUE and D2D groups varies, owing to changing interference situation; the transmit power of each UE will also be impacted. On the contrary, if the transmit power of each user alters, so does the interference condition between cochannel CUE and
D2D groups, which directly impacts the resource allocation result. Consequently, the above optimization problem is a mixed integer nonlinear programming (MINLP) problem which is known to be NP-hard and there are no efficient polynomial-time solutions [36]. Moreover, the complexity may also increase exponentially with the problem size. A possible solution might be using the bipartite matching based optimal resource allocation scheme proposed in [37]. However, such solution is actually based on exhaustive searching, which results in high computational complexity. In order to deal with this challenging problem, we propose a heuristic resource allocation algorithm so as to provide an effective solution.

According to expression and constraints formulated in (16), in order to improve energy efficiency of the D2D multicast group, we have to either decrease transmit power or to increase the aggregate transmission rate. It can be easily observed that optimization problem is a nonlinear constraint optimization problem. Hence, it is difficult to obtain the solution directly. In the following section, we solve the overall EE optimization problem by providing a heuristic resource allocation algorithm. We first consider increasing the numerator of expression (16). From the resource allocation point of view, if we want to improve aggregate D2D throughput, higher values of SINR are desirable. From constraints (16a)-(16e), we can deduce that smaller $G_{n, m}^{\mathrm{C} 2 \mathrm{D}}$ represents less interference from cochannel CUE $n$ to D2D group $m$, which results in higher $\gamma_{m, n}^{\mathrm{D} 2 \mathrm{D}}$ and D2D throughput. Hence, the basic idea of the proposed heuristic scheme is to pick up the CUE that generates less interference to cochannel D2D group. To achieve that target, we build a CSI matrix 
(1) $\mathscr{M}$ : List of D2D groups

(2) $\mathcal{N}$ : List of CUE items

(3) Build matrix $\mathbf{G}_{\mathrm{M} \times \mathrm{N}}^{\mathrm{C} 2 \mathrm{D}}$ according to (17)

(4) $j=1$

(5) while $\mathscr{M} \neq \varnothing$ and $j \leq M$ do

(6) $\quad m=\arg \min G_{i, j}^{\mathrm{C} 2 \mathrm{D}}, i=1,2, \ldots, N$

(7) Find $P_{m}^{\mathrm{D} 2 \mathrm{D}_{m}}$ and $P_{n}^{\mathrm{CUE}}$ from (18)

(8) if $P_{m}^{\mathrm{D} 2 \mathrm{D}_{m}} \leq P_{\max }^{\mathrm{DUE}}{ }^{n}$ and $P_{n}^{\mathrm{CUE}} \leq P_{\max }^{\mathrm{CUE}}$ then

(9) D2D group $m$ share resource with CUE $n$,

(10) Substitute $P_{m}^{\mathrm{D} 2 \mathrm{D}_{m}}$ and $P_{n}^{\mathrm{CUE}}$ into maximization problem built in (16), find the minimum transmit power so as to solve the overall energy-efficiency problem for D2D groups,

(11) $j=j+1$;

(12) else

(13) $j=j+1$;

(14) end if

(15) end while

Algorithm 1: An energy-aware resource and power allocation scheme for D2D multicast transmission.

$\mathbf{G}_{\mathbf{M} \times \mathbf{N}}^{\mathbf{C 2 D}}$, whose elements are the estimated CSI information of each D2D group from each CUE, respectively, where $\mathbf{G}_{\mathbf{M} \times \mathbf{N}}^{\mathbf{C 2 D}}$ is expressed as

$$
\mathbf{G}_{\mathbf{M} \times \mathbf{N}}^{\mathbf{C} 2 \mathrm{D}}=\left[\begin{array}{c}
G_{1,1}^{\mathrm{C} 2 \mathrm{D}}, G_{2,1}^{\mathrm{C} 2 \mathrm{D}}, \ldots, G_{N, 1}^{\mathrm{C} 2 \mathrm{D}} \\
G_{1,2}^{\mathrm{C} 2 \mathrm{D}}, G_{2,2}^{\mathrm{C} 2 \mathrm{D}}, \ldots, G_{N, 2}^{\mathrm{C} 2 \mathrm{D}} \\
\vdots \\
G_{1, M}^{\mathrm{C} 2 \mathrm{D}}, G_{2, M}^{\mathrm{C} 2 \mathrm{D}}, \ldots, G_{N, M}^{\mathrm{C} 2 \mathrm{D}}
\end{array}\right] .
$$

In matrix $\mathbf{G}_{\mathbf{M} \times \mathbf{N}}^{\mathrm{C2}}$, each row stands for the CSI information from cochannel CUE to corresponding DUE multicast group. We assume that each CUE sends the channel measurement information between itself and D2D receiver through control channel to BS. As a result, such information can be gathered at the BS side. Secondly, we find the minimum $G_{n, m}^{\mathrm{C} 2 \mathrm{D}}, n \in \mathcal{N}$ and $m \in \mathscr{M}$, in each row so as to pair up the CUE which brings the least interference to D2D multicast group. By doing the same procedure for each D2D multicast group, the optimal CUE and D2D multicast group can be decided.

After picking out the CUE for each D2D group, the next step is to decrease the denominator of expression (16). This is interpreted as to calculate the minimum transmit power of each CUE and D2D group, which also satisfies the minimum SINR threshold requirement. Suppose CUE $n$ shares cellular resources with D2D group $m$ after resource pairing process. By using constraints (13) and (14) and substituting $\gamma_{n}^{\mathrm{CUE}}$ and $\gamma_{m, n}^{\mathrm{D} 2 \mathrm{D}}$ from expressions (8) and (9) respectively, we can obtain the transmit power of D2D group and CUE which can guarantee reliable transmission as follows:

$$
\begin{aligned}
P_{m}^{\mathrm{D} 2 \mathrm{D}_{m}} & =\max _{\forall i, k \in m, n \in \mathcal{N}} \frac{\xi_{\min }^{\mathrm{D} 2 \mathrm{D}} \xi_{\min }^{\mathrm{CUE}} G_{n, k}^{\mathrm{C} 2 \mathrm{D}} \sigma_{n}^{2}+\xi_{\min }^{\mathrm{D} 2 \mathrm{D}} G_{n, \mathrm{BS}}^{\mathrm{CUE}} \sigma_{n}^{2}}{G_{i, k}^{\mathrm{D} 2 \mathrm{D}} G_{n, \mathrm{BS}}^{\mathrm{CUE}}-\xi_{\min }^{\mathrm{D} 2 \mathrm{D}} \xi_{\min }^{\mathrm{CUE}} G_{n, k}^{\mathrm{C} 2 \mathrm{D}} G_{i, \mathrm{BS}}^{\mathrm{D} 2 \mathrm{C}}}, \\
P_{n}^{\mathrm{CUE}} & =\max _{\forall i, k \in m, n \in \mathcal{N}} \frac{\xi_{\min }^{\mathrm{D} 2 \mathrm{D}} \xi_{\min }^{\mathrm{CUE}} G_{i, \mathrm{BS}}^{\mathrm{D} 2 \mathrm{C}} \sigma_{n}^{2}+\xi_{\min }^{\mathrm{CUE}} G_{i, k}^{\mathrm{D} 2 \mathrm{D}} \sigma_{n}^{2}}{G_{i, k}^{\mathrm{D} 2 \mathrm{D}} G_{n, \mathrm{BS}}^{\mathrm{CUE}}-\xi_{\min }^{\mathrm{D} 2 \mathrm{D}} \xi_{\min }^{\mathrm{CUE}} G_{n, k}^{\mathrm{C} 2 \mathrm{D}} G_{i, \mathrm{BS}}^{\mathrm{D} 2 \mathrm{C}}} .
\end{aligned}
$$

Combined with the power constraint given in (15), if the calculated transmit power of both CUE and DUE obtained in (18) is less than the maximum allowed transmission power, we choose CUE $n$ as the resource sharing partner for D2D group $m$. By substituting (18) into the object function (16), we can determine the minimum transmit power of different D2D multicast group so as to maximize the overall energy efficiency of D2D groups. On the other hand, if the calculated transmit power of both CUE and DUE is larger than the maximum allowed power constraints, then CUE $m$ will be removed from the list, and we will try the next available CUE. The pseudocode of the proposed energyaware resource allocation and power control algorithm is given in Algorithm 1.

In the proposed scheme, in order to maximize the energy efficiency of D2D multicast communication, we first search for the optimal resource pairing relationship between CUE and D2D groups, which leads to increased total throughput of D2D multicast transmission. Then, by taking the minimum SINR requirement of both CUE and D2D groups into consideration, we find out the minimum allowed transmit power of $\mathrm{D} 2 \mathrm{D}$ multicast groups to as to maximize the total energy efficiency. Hence, the maximization problem built in expression (16) will be solved $M$ times in the worst case, where the complexity of the proposed scheme is $\mathbf{O}(M N)+\mathbf{O}\left(M \times f\left|\mathscr{D}_{m}\right|\right)$. Here, $f\left|\mathscr{D}_{m}\right|$ represents the size of each D2D multicast group. We can see that, compared with conventional exhaustive searching based method [37] (where the complexity is $\mathbf{O}\left(M^{3}\right)+\mathbf{O}\left(M \times N \times f\left|\mathscr{D}_{m}\right|\right)$ ), our proposed heuristic resource allocation algorithm can considerably reduce the computational complexity.

After the above resource and power allocation scheme have been carried out for D2D multicast groups, $\mathrm{CH}$ in each multicast group can energy-efficiently distribute message to its group members. However, the energy condition of the $\mathrm{CH}$ still needs to be taken care of. More specifically, since $\mathrm{CH}$ serves as a source node and disseminates information to 
neighboring members of the same community, it actually sacrifices its own energy and storage resource to benefit others. This means $\mathrm{CH}$ will consume its energy more quickly than other group members, which results in its early energy depletion. To that end, the energy consumption issue becomes a crucial problem in the clustered based multicast transmission scenarios. In order to reduce energy consumption of the $\mathrm{CH}$ as well as maintain equal energy consumption of each group member, DUE within multicast group should act as $\mathrm{CH}$ in turn. However, unlike the conventional clustering strategies in ad hoc network which usually omit the potential social relationship among users [38], we need to incorporate both energy information and social information in the $\mathrm{CH}$ selection and update algorithm. In the next section, we will deal with $\mathrm{CH}$ update algorithm from the aspects of energy efficiency and social relationship.

\section{Social and Energy-Aware Cluster Head Updated Algorithm}

Generally speaking, the energy consumption of each UE depends on transmission/reception time and bit rate [39]. In this work, we use the generic energy consumption model proposed in [40], where the energy consumption is formulated as $P(R) T . P(R)$ represents the energy consumed per second when transmitting or receiving with bit rate $R$ and $T$ is the time duration in seconds.

For D2D multicast communication, assume the transmission in the time domain is on a slot-to-slot basis, where the duration of each slot is $T$. Therefore, in each multicast transmission slot, the energy consumption of DUE $i$ that serves as a $\mathrm{CH}$ in $m$ th $\mathrm{D} 2 \mathrm{D}$ group will be

$$
E_{i}^{T x}=P_{m}^{\mathrm{D}^{2} \mathrm{D}_{m}}\left(r_{m}^{\mathrm{D} 2 \mathrm{D}}\right) T, \quad \forall i \in m .
$$

Similarly, for a DUE $k$ who acts as a multicast receiver in $m$ th D2D group, the energy consumption in each multicast transmission slot is given by

$$
E_{k}^{R x}=P_{k}^{\mathrm{D} 2 \mathrm{D}_{m}}\left(r_{m}^{\mathrm{D} 2 \mathrm{D}}\right) T, \quad \forall k \in \mathscr{D}_{m}
$$

where $P_{k}^{\mathrm{D}^{2} \mathrm{D}_{m}}\left(r_{m}^{\mathrm{D} 2 \mathrm{D}}\right)$ stands for the receiving energy consumption with bit rate $r_{m}^{\mathrm{D} 2 \mathrm{D}}$.

As a result, the remaining energy of a DUE $d$ which belongs to $m$ th D2D group is then expressed as

$$
E_{d}^{\mathrm{Re}}=W-\left\{E_{i}, E_{k}\right\}, \quad \forall i, d \in m, k \in \mathscr{D}_{m},
$$

where $W$ represents the initial energy. Suppose all DUE items in a D2D multicast group have the same energy level at the initial period. Since each DUE in the multicast group either acts as a $\mathrm{CH}$ or acts as a receiver. Consequently, we can calculate the remaining energy of each user according to (21). It can be inferred that the DUE that consumes less energy in the multicast period will survive longer. Hence the remaining energy, which implies the physical survival time of a particular DUE, is an essential index for the selection of a $\mathrm{CH}$. By introducing a threshold $\Gamma^{\text {Energy }}$ which gives the minimum energy requirement of a DUE, we can formulate a new set $A$ and pick out those DUE items that are capable of functioning as a $\mathrm{CH}$ based on physical remaining energy constraint

$$
\begin{array}{ll} 
& \mathrm{A}=\{\mathrm{DUE} d\} \\
\text { s.t. } & E_{d}^{\mathrm{Re}}>\Gamma^{\text {Energy }}, \quad \forall d \in m .
\end{array}
$$

According to expression (22), DUE items that have little remaining energy could not serve as a $\mathrm{CH}$ due to high energy consumption accompanied with D2D multicast transmission. Only those DUE items that have enough remaining energy will be held in set $A$.

On the other hand, unlike the existing ad hoc network where users have no social relationships and discover each other by sending probe request packets [41], in a D2D multicast scenario, users' social relations are long-term accumulated characteristics that facilitate the discovery of proper D2D peers. Accordingly, DUE items with similar interests tend to request the similar content, where multicast groups are formed by users with strong social ties. Instead of retrieving data from BS, DUE can receive the content from a $\mathrm{CH}$, who acts as a source node and disseminates the same information to neighbor users. Arguably, when considering $\mathrm{CH}$ updated algorithm, DUE with a high number of social ties or more important (i.e., influential) in the group is mostly preferred as a $\mathrm{CH}$. Hence, for DUE items that have strong social connections with other DUE, reliable D2D links can be established for data sharing. In order to determine the importance of DUE in social networks, we introduce the centrality metric [13]. Centrality metric measures the social influence of a DUE based on how well it connects the network, whereby a DUE with high centrality is considered more important to its social group community.

With reference to the social tie relationship built in Section 2, we can construct the social relationship indication matrix $\mathbf{H}_{m \times m}$ of $m$ th D2D group as

$$
\mathbf{H}_{m \times m}=\left[\begin{array}{c}
\omega_{1,1}, \omega_{1,2}, \ldots, \omega_{1, m} \\
\omega_{2,1}, \omega_{2,2}, \ldots, \omega_{2, m} \\
\vdots \\
\omega_{m, 1}, \omega_{m, 2}, \ldots, \omega_{m, m}
\end{array}\right] \text {; }
$$

here $\omega_{i, j}, \quad \forall i, j \in m$, represents the strength of the social relationship between DUE $i$ and $j$, where the value of $\omega_{i, j}$ can be obtained through (4).

In order to pick out a set of influential users who have stronger social ties among a D2D multicast group, we need to obtain the centrality metric of each user. Here, eigenvector centrality is used for detecting a set of influential users, which estimates the influence of users in the network by using eigenvector corresponding to the largest eigenvalue of the social relationship matrix of the network. Remember that eigendecomposition and singular value decomposition of matrix are two essential computations in linear algebra, which have been widely applied in engineering fields, such as signal processing and face recognition [42]. Given 


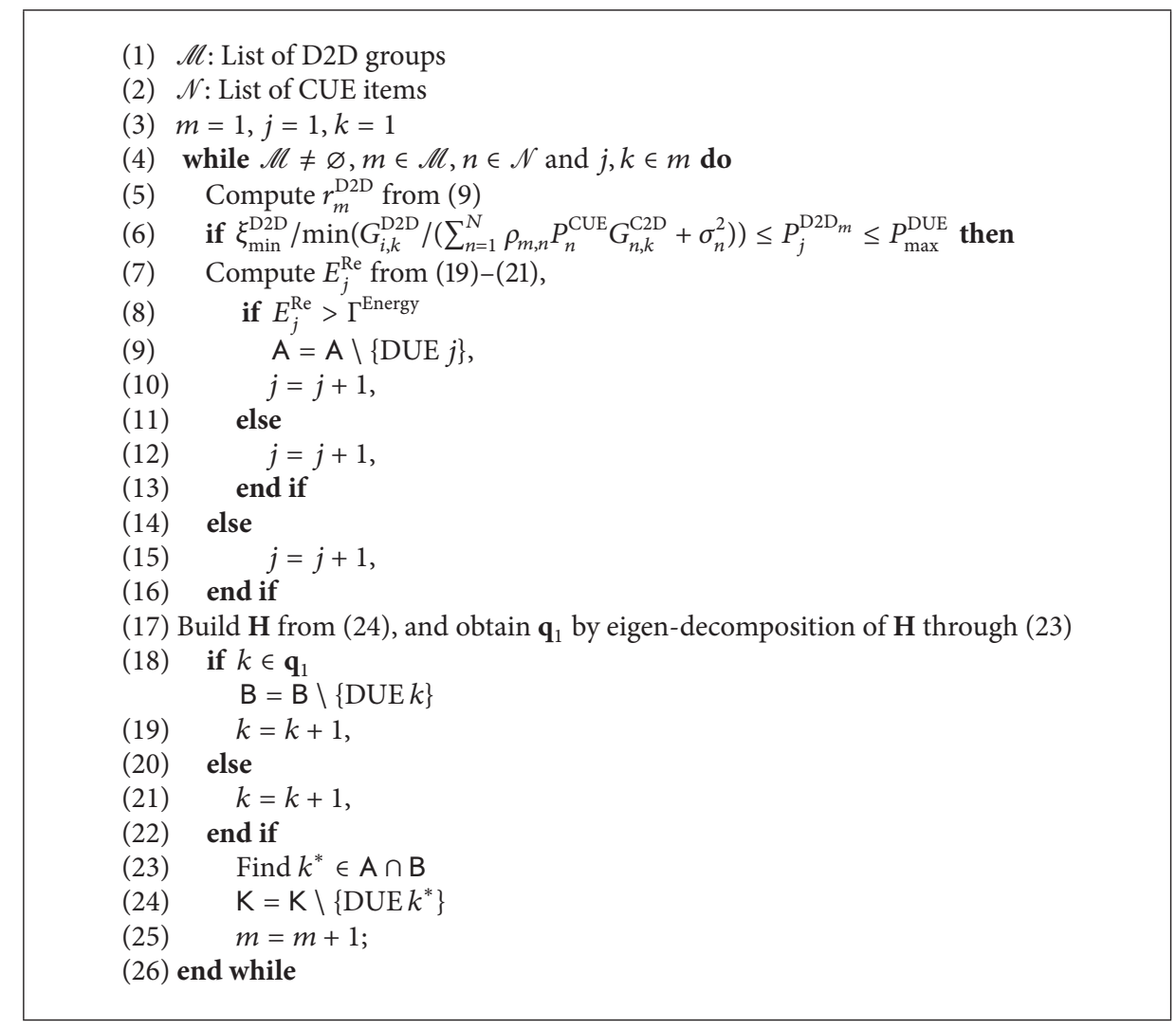

Algorithm 2: A social and energy-aware $\mathrm{CH}$ update scheme.

a $m \times m$ symmetric matrix $\mathbf{H}$, it can be diagonalized using its eigenvalues and eigenvectors as follows:

$$
\begin{aligned}
\mathbf{H} & =\mathbf{Q} \boldsymbol{\Lambda} \mathbf{Q}^{-1} \\
& =\left[\mathbf{q}_{1}, \mathbf{q}_{2}, \ldots, \mathbf{q}_{m}\right]\left(\begin{array}{ccc}
\lambda_{1} & \cdots & 0 \\
\vdots & \ddots & \vdots \\
0 & \cdots & \lambda_{m}
\end{array}\right)\left[\begin{array}{c}
\mathbf{q}_{1}^{T} \\
\mathbf{q}_{2}^{T} \\
\vdots \\
\mathbf{q}_{m}^{T}
\end{array}\right],
\end{aligned}
$$

where $\mathbf{Q}$ is a $m \times m$ orthonormal matrix whose columns are orthonormal eigenvectors of matrix $\mathbf{H}$ and $\boldsymbol{\Lambda}$ is a diagonal matrix containing the eigenvalues $\lambda_{1}, \ldots, \lambda_{m}$ of the matrix $\mathbf{H}$ sorted in descending order. Then the eigenvector centrality is basically eigenvector $\mathbf{q}_{1}$, which corresponds to the largest eigenvalues $\lambda_{1}$. Thus, we can build another set $B$ and obtain several most influential DUE items from eigenvector $\mathbf{q}_{1}$, which is given by

$$
\begin{array}{ll} 
& \mathrm{B}=\{\text { DUE } k\} \\
\text { s.t. } & k \in \mathbf{q}_{1} .
\end{array}
$$

Combining with the physical energy constraint as well as social centrality, we propose a social and energy-aware $\mathrm{CH}$ update strategy; the pseudocode is given in Algorithm 2.

In the proposed social and energy-aware $\mathrm{CH}$ update algorithm, the energy constraints, as well as social ties among users are taken into consideration. In particular, for a D2D multicast group, in order to select the appropriate $\mathrm{CH}$ who is capable of multicast transmission, the remaining energy is the primary factor that needs to be addressed. Although multicast may be beneficial for some users, $\mathrm{CH}$ who serves as source node may end at battery depletion. Only those DUE items that are powered with enough energy can serve as a $\mathrm{CH}$. On the other hand, social ties measure the strength with which a group of entities are related to each other. It is assumed that only those DUE items that have strong social connections among a group can be served as a trusted $\mathrm{CH}$. In order to determine such influential users, the concept of eigenvector centrality is introduced. By building the social relationship indication matrix and with the help of eigendecomposition, those users who correspond to the largest eigenvalues will be picked out.

Based on the energy constraint and social centrality, only those DUE items that have strong social connections in a D2D multicast group and also have enough energy are eligible to be selected as a $\mathrm{CH}$. Finally, since the new set $\mathrm{K}$ which comprises of potential CHs is built for a D2D multicast group, a new $\mathrm{CH}$ can be selected and updated for the original D2D multicast group for a certain period. However, accompanied with the $\mathrm{CH}$ update process, the cluster size and group member might also need to be updated. This is because when a new $\mathrm{CH}$ is selected, the social relationship between the new $\mathrm{CH}$ and each member might also change. Consequently, the proposed social tie based D2D multicast group formation 
TABLE 1: The simulation parameters.

\begin{tabular}{lc}
\hline Parameter & Value \\
\hline Cell radius & $400 \mathrm{~m}$ \\
Total UE number $(N)$ & 500,1000 \\
Spectrum bandwidth & $10 \mathrm{MHz}$ \\
Bandwidth of each channel & $180 \mathrm{KHz}$ \\
Path loss factor $(\alpha)$ & 4 \\
& Log-normal fading \\
Shadowing & with standard \\
& deviation of $8 \mathrm{~dB}$ \\
Noise power spectrum density $\left(\sigma^{2}\right)$ & $-174 \mathrm{dBm} / \mathrm{Hz}$ \\
Minimum SINR threshold $\left(\xi_{\min }^{\mathrm{D} 2 \mathrm{D}}, \xi_{\min }^{\mathrm{CUE}}\right)$ & $10 \mathrm{~dB}$ \\
Maximum transmit power of $\mathrm{UE}\left(P_{\max }^{\mathrm{CUE}}, P_{\max }^{\mathrm{DUE}}\right)$ & $\mathrm{CUE}: 23 \mathrm{dBm}$ \\
Number of D2D groups $(M)$ & DUE: $20 \mathrm{dBm}$ \\
Weight coefficient $(\omega)$ & 8 \\
Social threshold $\left(\Gamma_{d_{i}}^{\text {So }}\right)$ & {$[0,1]$} \\
D2D group radius $(r)$ & 1 \\
UE transmission range & $30 \sim 90 \mathrm{~m}$ \\
\hline
\end{tabular}

strategy might also be triggered by the $\mathrm{CH}$ update process. If $\mathrm{K}=\varnothing$, this means there is no available $\mathrm{CH}$ in current period. Hence, the $\mathrm{CH}$ update process should be terminated.

\section{Simulations}

In this section, we present numerical results to illustrate the performance of our proposed strategy. We consider the scenario such as sports stadium or concert, where hundreds even thousands of people are gathered in a small region who are eager to express their real-time opinion or share photos with their friends [43]. Under such situation, social-aware based D2D multicast transmission can be exploited to retrieve the BS from excessive load burden. The simulated network is contained in a $400 \mathrm{~m} \times 400 \mathrm{~m}$ square area, where all the users are randomly distributed in this area according to uniform distribution. The initial cluster heads who want to start D2D multicast transmissions are also randomly distributed among users. The distance based path loss and shadowing fading are considered for transmission channel. For resource allocation and sharing method, we still take sharing uplink cellular resource as an example. Assume that all the available resource is divided into channels, and each accessed UE is allocated with one channel at each scheduling slot. According to [40], the energy consumed per unit time can be considered to be almost constant for various transmission bit rates using adaptive rate control. Following these studies, we set the energy consumption value of UE as $P_{m}^{\mathrm{D} 2 \mathrm{D}_{m}}\left(r_{m}^{\mathrm{D} 2 \mathrm{D}}\right)=$ 1.8 Joule/sec and $P_{k}^{\mathrm{D}^{2} \mathrm{D}_{m}}\left(r_{m}^{\mathrm{D} 2 \mathrm{D}}\right)=0.925 \mathrm{Joule} / \mathrm{sec}$. Other related simulation parameters are listed and shown in Table 1.

Note that, in the following simulation results, we first evaluate the performance of the proposed social tie based D2D multicast group formation scheme. Then based on the $\mathrm{D} 2 \mathrm{D}$ multicast group formation results, we compare

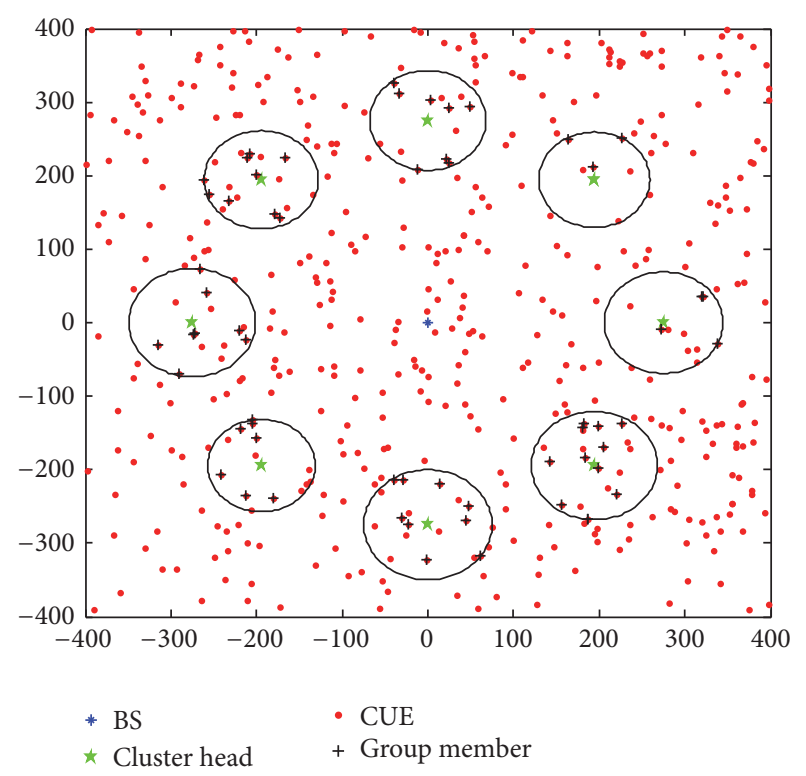

FIGURE 2: Initial multicast group formation results.

the performance of our proposed energy-aware resource and power allocation scheme with three other different resource allocation algorithms. The first one is the QoS-aware resource allocation scheme proposed in [20], which aims at minimizing the interference among D2D multicast group and CUE through resource allocation. Moreover, [20] assumes both CUE and D2D group transmit at the maximum power. The second one is the energy-efficient scheme proposed in [15], which aims at improving the energy efficiency multicast transmission through proper power control. The power control principle is similar to our proposed scheme. The third one is the cluster based scheme proposed in [28], which employs social information to facilitate file transfer problem. The proposed scheme first utilizes social information to help form D2D multicast cluster and then focuses on using different duplex approach to support asynchronous reception and transmission at the cluster head. In the absence of resource allocation scheme between D2D group and CUE, we assume that the resource reuse relationship between D2D cluster and CUE in [27] is chosen randomly while power control method is not applied. For clarity, our proposed energy-aware resource and power allocation scheme is referred to as the "proposed energy-aware" scheme. Finally, we will illustrate the performance of our proposed social and energy-aware $\mathrm{CH}$ update scheme.

The social tie based D2D multicast group formation result in Figure 2 shows that the multicast group formation heavily depends on both the physical and social domain information. We assume there are totally $500 \mathrm{UE}$ items randomly distributed in the simulation area. According to our proposed strategy, when a $\mathrm{CH}$ prepares to initiate a $\mathrm{D} 2 \mathrm{D}$ multicast transmission, it has to first build a neighbor set, where the neighbor DUE items that are located within the maximum transmission range can join the multicast group from the physical domain point of view. Then, considering the social relationship among users, those DUE items that have strong 


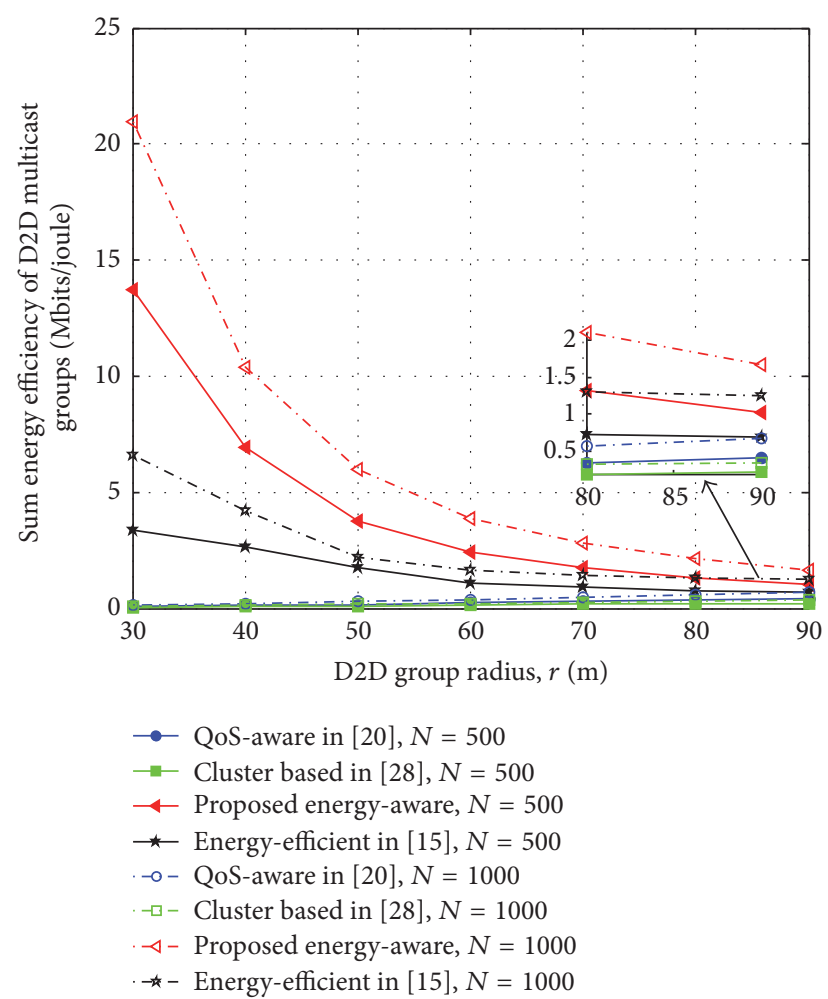

FIGURE 3: D2D group energy efficiency versus D2D group radius.

social connections with the $\mathrm{CH}$ will be retained in the $\mathrm{D} 2 \mathrm{D}$ multicast group where unfamiliar DUE items are excluded from the multicast transmission. Interestingly, as can be observed, the group radius and the number of group users in each D2D multicast group are quite different. This is because the group radius of each D2D multicast group is determined by the group member who is farthest from the $\mathrm{CH}$. As a result, according to the physical user distribution as well as social tie strength among users, different multicast group is formulated by the $\mathrm{CH}$ in a distributed way. Compared with the cluster formation method proposed in [44] which is carried out in a centralized way by the BS, our proposed social tie based group formation method can be adaptable to changing user numbers and social relationships, where a multicast group is formulated based on both network and social dimension requirement.

Figure 3 illustrates the sum energy efficiency of the D2D multicast group with the variation of the $\mathrm{D} 2 \mathrm{D}$ group radius. Unlike the work in [36] which defines the D2D cluster distance as the distance between two DUE pairs, here we define the D2D group distance as the distance between the $\mathrm{CH}$ and the group user who is farthest from $\mathrm{CH}$. From Figure 3 we can see that, with the increase of the radius of the D2D group, the EE performance of our proposed scheme and the scheme in [15] is better than that of the scheme in $[20,28]$. This is because the channel gain of the D2D link decreases when the group radius increases. Hence, more transmission power is required for the $\mathrm{D} 2 \mathrm{D}$ groups so as to satisfy the SINR constraint. Both our proposed scheme and the scheme in [15] adopt power control method which adaptively

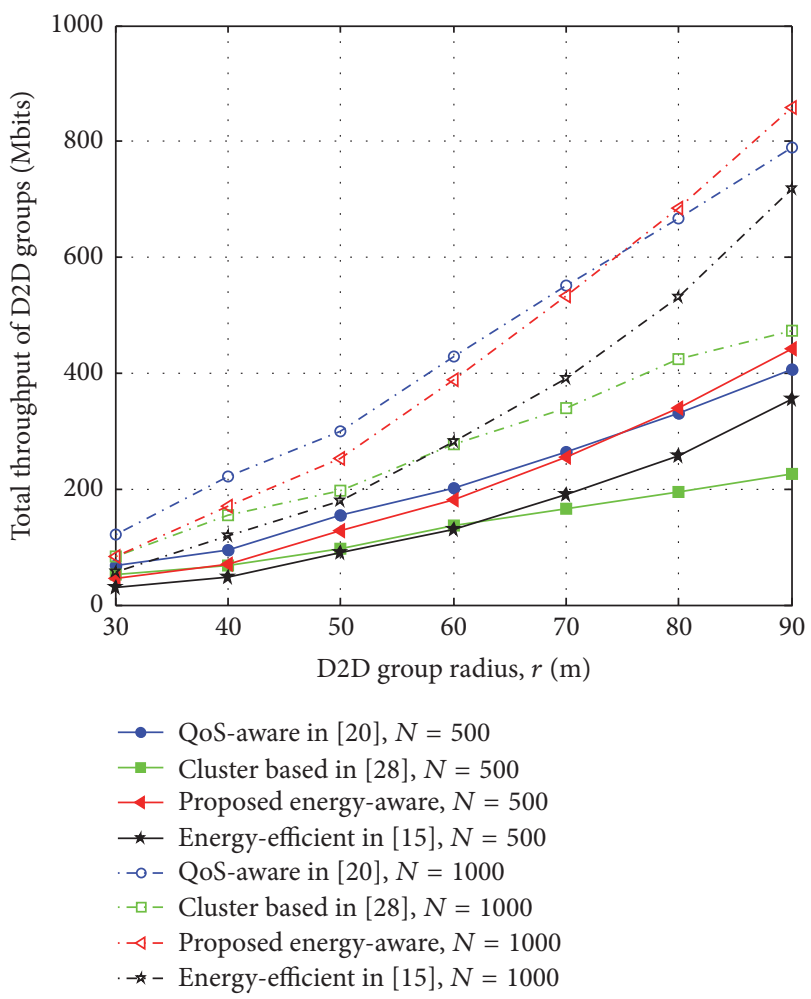

Figure 4: Total throughput of D2D multicast groups versus D2D group radius.

adjusts the transmit power according to changing channel conditions, while the schemes in $[20,28]$ always transmit at the maximum power. With the increase of D2D group radius, our proposed scheme will gradually increase the transmit power which results in decreasing energy efficiency of the D2D group. On the other hand, we can see the EE performance of $[20,28]$ slight increases with the increase of $\mathrm{D} 2 \mathrm{D}$ group radius. This is because owing to the increased coverage of the $\mathrm{CH}$, more UE might locate in the coverage of D2D multicast groups and become group members. Recall that, in expression (16), EE is expressed as the sum of the transmission rate for all D2D multicast groups to the sum of total transmit power. When there are more group members in each D2D multicast group, the total D2D throughput might also increase despite the deteriorating channel condition of D2D links. Compared with the scheme in [15], our proposed energy-aware resource allocation method is based on minimizing the interference of $\mathrm{D} 2 \mathrm{D}$ groups suffering from CUE. Hence, the interference from CUE to cochannel D2D receivers will be decreased, which improves the channel gain of D2D links. Furthermore, it is shown that when there are more UE items in the considered scenarios ( $N$ varies), there will be more UE located in each D2D multicast groups, which contributes to larger EE due to increased total transmission rate of all D2D multicast groups.

Figure 4 compares the total throughput of D2D multicast groups of the proposed scheme with three other schemes. From the figure, we can see that the total throughput performance of the proposed scheme is initially inferior to that 
of [20] and gradually outperforms other schemes with the increase of D2D group radius. The reason is twofold. Firstly, after D2D multicast groups have been formed, our proposed energy-aware resource and power allocation scheme aims at maximizing the EE performance of the $\mathrm{D} 2 \mathrm{D}$ groups, which will adopt a smaller transmit power when the radius of the D2D multicast group is small. This explains why the total throughput of our proposed scheme is smaller compared with that of the scheme in [20], which always adopt the maximum transmit power. However, with the increase of D2D group radius, our proposed scheme will gradually increase the transmit power of DUE items while decreasing the transmit power of cochannel CUE in order to ensure reliable D2D multicast transmission, which leads to increased total throughput. On the other hand, with the increase of D2D group radius, since both CUE and D2D groups in [20] always transmit at the maximum power, the interference between cochannel CUE and D2D groups will also severely impact the channel gain of the D2D link, which leads to a slow increase of total throughput. Secondly, compared with schemes in [15, 28], the resource reuse relationship of our proposed scheme is based on minimizing the interference of D2D links suffering from CUE. As a result, the CUE which brings the least interference to cochannel D2D multicast group will be paired up with the D2D multicast group in our proposed scheme, while for schemes in $[15,28]$, the resource reuse relationship between CUE and D2D groups are randomly chosen. This explains why our proposed scheme has better performance compared with these schemes. Similarly, it is shown that the sum throughput of all four algorithms increases with the increase of user numbers.

The impact of energy efficiency on total throughput of D2D multicast groups is shown in Figure 5. It can be observed that EE performance of both our proposed scheme and the scheme in [15] decreases with the increase of total throughput. This is explained as when the total throughput increases, according to our proposed power control scheme, the transmit power also increases, which contributes to decreased energy efficiency. However, for schemes [20, 28 ], since UE always adopts the maximum power, the EE performance slightly increases with the increase of total throughput. Moreover, when the total UE numbers remain the same, the adopted heuristic resource allocation algorithm in our proposed scheme allocates the better cellular resource to D2D groups based on minimizing the interference of D2D groups while at the same time decreasing the transmit power of cochannel CUE, which contributes to improved EE performance of D2D groups.

Figure 6 plots the average SINR of D2D multicast group with different $\mathrm{D} 2 \mathrm{D}$ group radius. We assume there are totally $500 \mathrm{UE}$ items randomly distributed in the simulation area. From the figure, we can infer that, for the schemes which include power control scheme, such as our proposed algorithm and scheme in [15], the SINR distribution does not obviously decrease with the increase of D2D group radius. This is because according to the changing channel conditions and resource reuse relationships, the power control method joint adjusts the transmission power of both D2D group and CUE, which ensures reliable D2D multicast transmission of

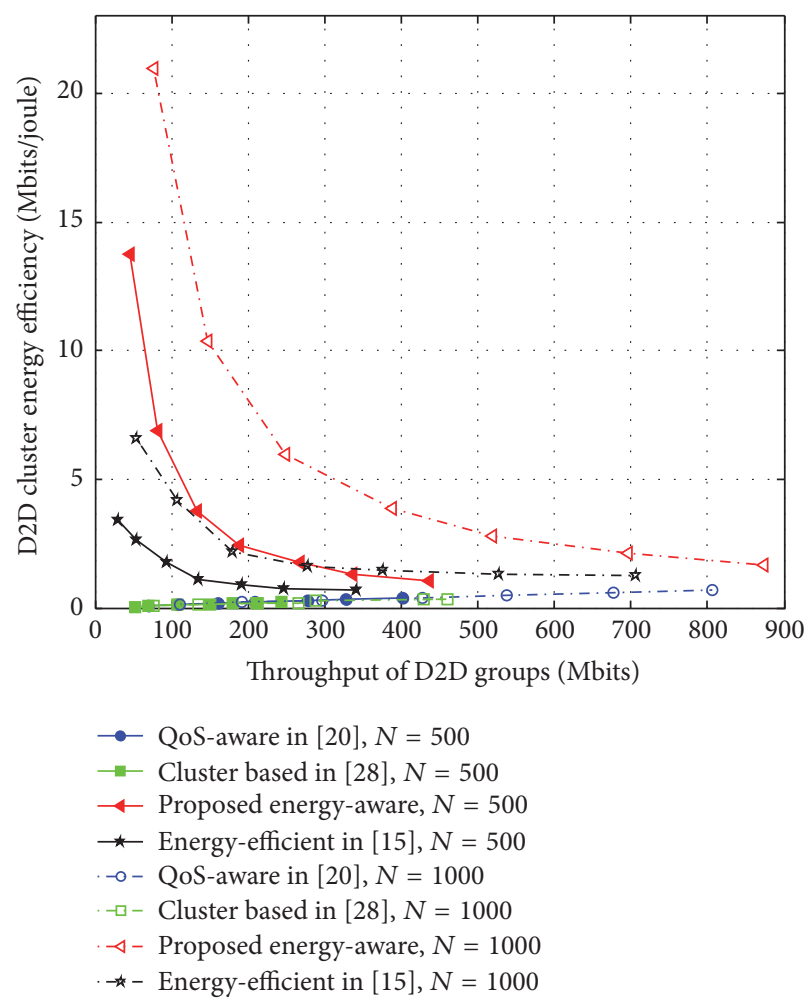

FIGURE 5: D2D group energy efficiency versus throughput.

each D2D multicast group. On the contrary, for the algorithms which do not incorporate power control schemes, the minimum SINR requirement of a D2D group might not be met with the increase of $\mathrm{D} 2 \mathrm{D}$ group radius. This is explained as the total transmission rate of a D2D multicast group actually depends on the group user with the worst channel condition, as shown in expression (9). When the group radius gradually becomes larger, the SINR of the group user who is located at most disadvantageous place decreases at the same time. Since both the schemes in $[20,28]$ suggest keeping the maximum transmit power for D2D group and CUE at all the times, the QoS requirement in terms of minimum SINR constraint cannot be guaranteed when the channel quality becomes worse. Moreover, our proposed energyaware resource and power allocation scheme outperforms the scheme in [15] owing to the adopted heuristic resource allocation scheme between D2D group and CUE, which decrease cochannel interference of D2D groups coming from CUE.

Figure 7 demonstrates the average energy efficiency of D2D group with a variant number of users in each $\mathrm{D} 2 \mathrm{D}$ group. The D2D group radius is $50 \mathrm{~m}$, where there are totally 500 UE items. In order to reveal the relationship between group formation method and energy efficiency, we also consider the situation when D2D multicast group is formulated in a centralized way. With reference to [44], the centralized D2D multicast group formation is achieved by the BS according to the physical constraints only. More specifically, group users who are located within the transmission range of the $\mathrm{CH}$ will join in the $\mathrm{D} 2 \mathrm{D}$ multicast group where social relations among group members are 


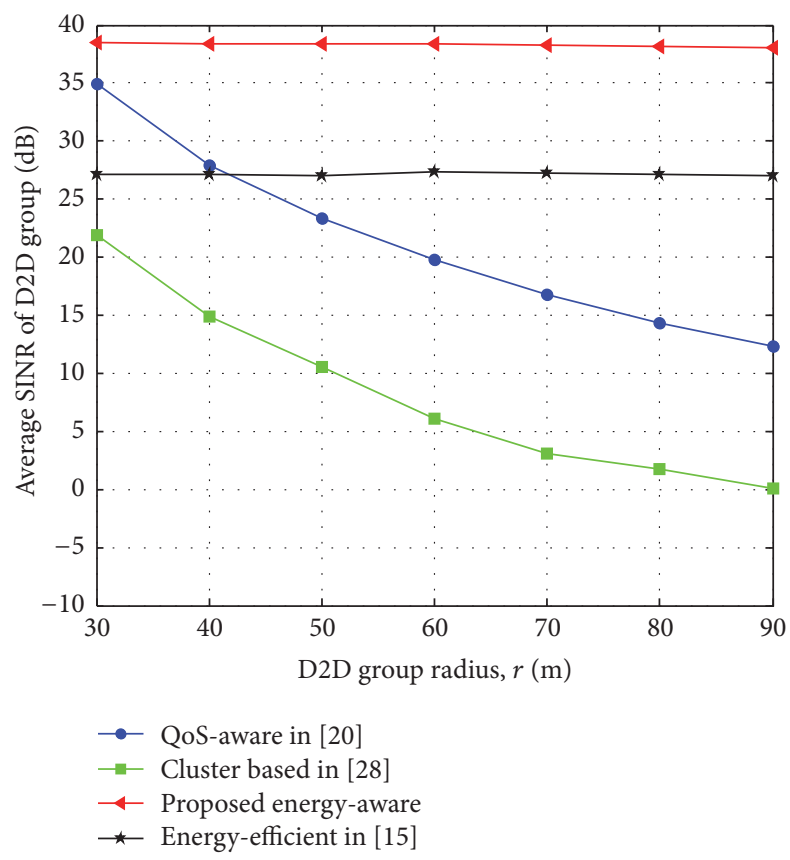

Figure 6: Average SINR of D2D multicast group versus D2D group radius.

omitted. In Figure 7, our proposed social tie based D2D multicast group formation is referred to as "social tie based," while the centralized D2D multicast group formation is referred to as "centralized." As can be observed from the figure, with the increase of group users in each D2D multicast group, the average energy efficiency of the $\mathrm{D} 2 \mathrm{D}$ group also increases. This is because when there are more group users within the D2D multicast groups, the sum of the transmission rate will increase, which contributes to increased energy efficiency of each D2D group. Moreover, the performance of our proposed resource allocation scheme plus social tie based multicast group formation algorithm is better than other algorithms. This is because our proposed group formation scheme first forms D2D multicast group based on both physical and social domain information. Those users who are both physically within the transmission range of the $\mathrm{CH}$ and also socially familiar with each other will join the same D2D multicast group. This helps to exclude unnecessary and insecure transmission between the $\mathrm{CH}$ and $\mathrm{D} 2 \mathrm{D}$ receivers in each multicast group, which further decreases the transmit power of the $\mathrm{CH}$. Then, our proposed energy-aware resource allocation scheme considers both resource allocation and power allocation method so as to maximize the total energy efficiency, which in turn results in increased average energy efficiency in each D2D multicast group. From the figure, we can infer that our proposed energy-aware resource allocation and power control method further improves energy efficiency of D2D multicast transmission by using the knowledge of social tie strength among multicast group users.

In Figure 8, the effect of the social threshold on different resource allocation schemes is illustrated. The D2D group radius and total UE numbers are the same as Figure 7, where our proposed social tie based D2D multicast group

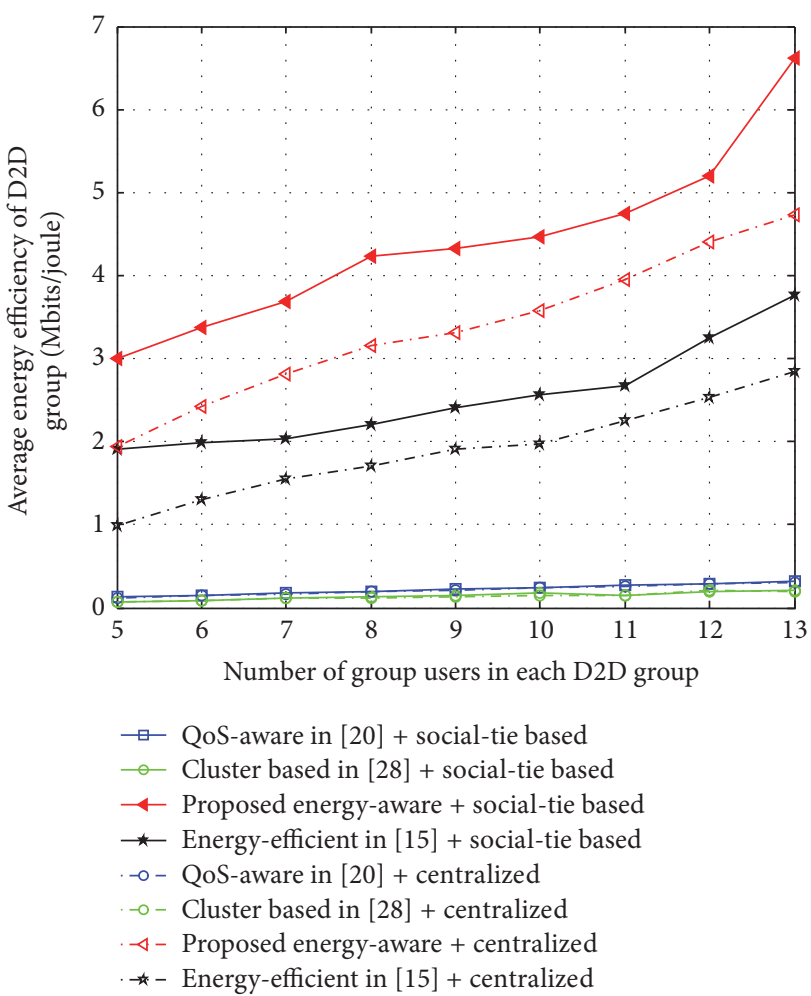

FIGURE 7: Average D2D group energy efficiency versus number of group users.

formation is applied before the resource allocation process. From the figure, it is seen that increasing the social threshold leads to decreasing average energy efficiency of D2D group. This phenomenon is explained since all considered resource allocation schemes are carried out after D2D multicast group formation. When the social threshold becomes tight (e.g., $\Gamma_{d_{i}}^{\text {So }}$ becomes larger), it actually limits the chances for a UE to become a D2D group member and join the multicast transmission owing to trust issues resulting from weak social connections. As a result, fewer UE items will become D2D multicast group members, which leads to decreased total transmission rate of $\mathrm{D} 2 \mathrm{D}$ multicast transmission.

Figure 9 plots the D2D multicast group cluster head update results after a certain simulation time. Compared with the initial group formation result shown in Figure 2, we can see that the CHs in some multicast group have been updated and the group members have also been changed. This result is explained as according to our proposed social and energy-aware $\mathrm{CH}$ update algorithm, a new $\mathrm{CH}$ who has enough energy might be selected after a certain time period so as to save the remaining energy of the initial $\mathrm{CH}$. Moreover, the newly selected $\mathrm{CH}$ should also have high social centrality among the D2D multicast group. According to both physical energy constraint and social centrality, only those group members who have strong social connections in a D2D multicast group and also have enough remaining energy are eligible to act as a $\mathrm{CH}$. This explains why some multicast groups have updated $\mathrm{CH}$ while some are not. After the new $\mathrm{CH}$ is selected, the social relationship between the new $\mathrm{CH}$ 


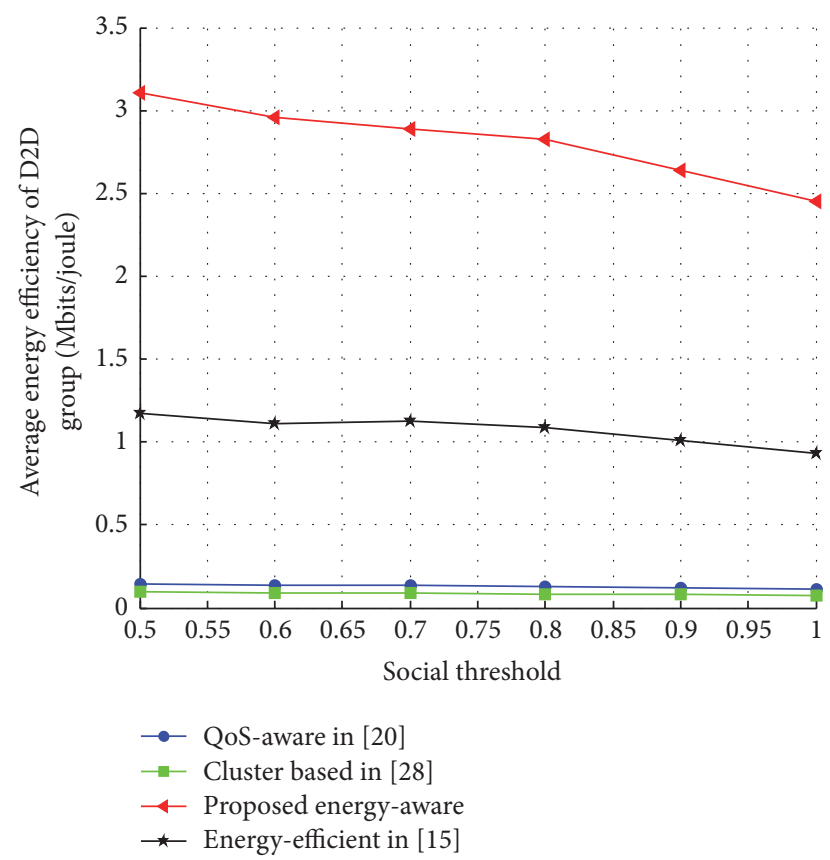

Figure 8: Average D2D group energy efficiency versus social threshold.

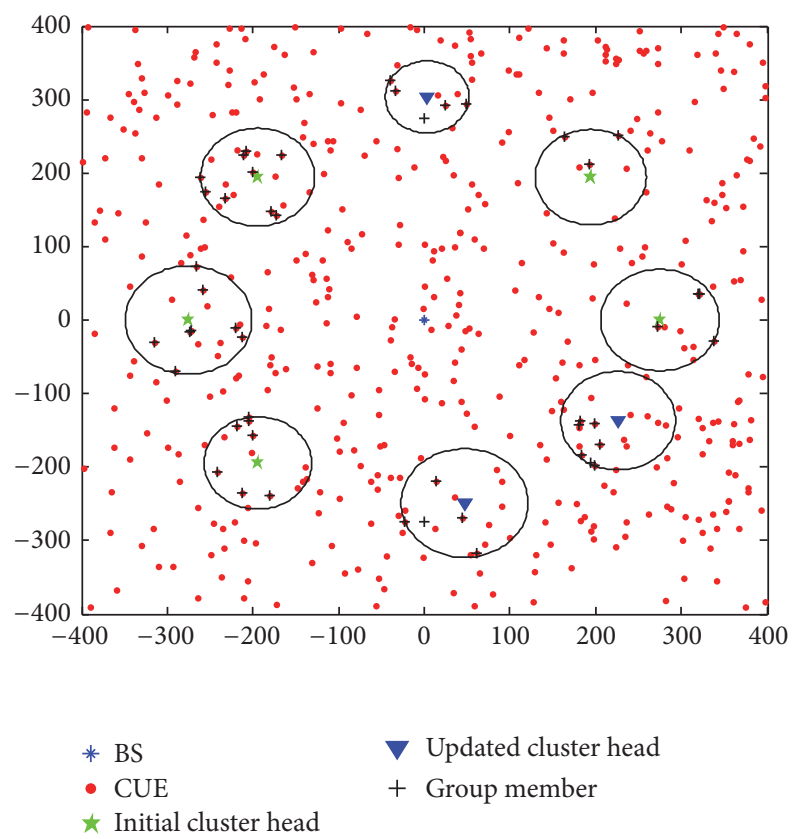

FIGURE 9: D2D multicast group CH updated results.

and each group member might also change. Consequently, the proposed social tie based D2D multicast group formation strategy might also be triggered which attributes to changed group users.

\section{Conclusion}

In this paper, we have investigated energy-efficient cluster formation and resource allocation algorithms for multicast
D2D communication underlaying a cellular network. By incorporating social knowledge, we first deal with D2D multicast group formation strategy from both physical and social domain constraint. Then we aim at maximizing the overall energy efficiency of D2D multicast groups through appropriate resource allocation and power control scheme, while considering the SINR requirements of both CUE and D2D groups. A heuristic resource and power control algorithm is then proposed to solve above energy efficiency problem with less complexity. Finally, by taking into account the limited battery capacity of mobile users, we propose a social and energy-aware cluster head update algorithm, which incorporates both the energy constraint in the physical domain and social centrality measurement in the social domain. It is shown by simulation that the energy efficiency of D2D multicast groups with the proposed scheme can be improved significantly compared with conventional multicast schemes. Furthermore, by exploiting social domain information in the physical domain underlay D2D multicast transmission provides an energy-effective method to mitigate the burden of BS and improves network performance.

\section{Conflicts of Interest}

The authors declare that they have no conflicts of interest.

\section{Acknowledgments}

This work is supported by the National Natural Science Foundation of China (no. 61501371, no. 61271276), National 863 High Tech R\&D Program of China (Project no. 2014AA01A703), and the International Exchange and Cooperation Projects of Shaanxi Province (Project no. 2016KW046).

\section{References}

[1] D. Evans, The Internet of Things: How the Next Evolution of the Internet Is Changing Everything, Cisco Internet Business Solutions Group (IBSG), 2011.

[2] IMT-2020 (5G) Promotion Group, White Paper on 5G Wireless Technology Architecture, 2015, http://www.3gpp.org/technologies/presentations-white-papers.

[3] M. Tehrani, M. Uysal, and H. Yanikomeroglu, "Device-todevice communication in 5G cellular networks: challenges, solutions, and future directions," IEEE Communications Magazine, vol. 52, no. 5, pp. 86-92, 2014.

[4] A. Gupta and R. K. Jha, "A survey of 5G network: architecture and emerging technologies," IEEE Access, vol. 3, pp. 1206-1232, 2015.

[5] L. Wei, R. Q. Hu, Y. Qian, and G. Wu, "Enable device-to-device communications underlaying cellular networks: challenges and research aspects," IEEE Communications Magazine, vol. 52, no. 6, pp. 90-96, 2014.

[6] Technical Specification Group Services and System Aspects, "Study on architecture enhancements to support Proximity Services (ProSe)," 3GPP TR 23.703 v0.5, Rel 12, 2013.

[7] M. Condoluci, L. Militano, G. Araniti, A. Molinaro, and A. Iera, "Multicasting in LTE-A networks enhanced by device-todevice communications," in Proceedings of the IEEE Globecom 
Workshops (GC '13), pp. 567-572, Atlanta, Ga, USA, December 2013.

[8] X. Lin, R. Ratasuk, A. Ghosh, and J. G. Andrews, "Modeling, analysis, and optimization of multicast device-to-device transmissions," IEEE Transactions on Wireless Communications, vol. 13, no. 8, pp. 4346-4359, 2014.

[9] L. Xu, C. Jiang, Y. Shen, T. Q. Quek, Z. Han, and Y. Ren, "Energy efficient D2D communications: a perspective of mechanism design," IEEE Transactions on Wireless Communications, vol. 15, no. 11, pp. 7272-7285, 2016.

[10] M. Ali, S. Qaisar, M. Naeem, and S. Mumtaz, "Energy efficient resource allocation in D2D-assisted heterogeneous networks with relays," IEEE Access, vol. 4, pp. 4902-4911, 2016.

[11] O. Semiari, W. Saad, S. Valentin, M. Bennis, and H. V. Poor, "Context-aware small cell networks: how social metrics improve wireless resource allocation," IEEE Transactions on Wireless Communications, vol. 14, no. 11, pp. 5927-5940, 2015.

[12] X. Chen, B. Proulx, X. Gong, and J. Zhang, "Exploiting social ties for cooperative D2D communications: a mobile social networking case," IEEE/ACM Transactions on Networking, vol. 23, no. 5, pp. 1471-1484, 2015.

[13] E. Datsika, A. Antonopoulos, N. Zorba, and C. Verikoukis, "Green cooperative device-to-device communication: a social-aware perspective," IEEE Access, vol. 4, pp. 3697-3707, 2016.

[14] T. Wang, Y. Sun, L. Song, and Z. Han, "Social data offloading in D2D-enhanced cellular networks by network formation games," IEEE Transactions on Wireless Communications, vol. 14, no. 12, pp. 7004-7015, 2015.

[15] N. Guan, Y. Zhou, H. Liu et al., "An energy efficient cooperative multicast transmission scheme with power control," in Proceedings of the IEEE Global Telecommunications Conference (GLOBECOM '11), pp. 1-5, Houston, Tex, USA, December 2011.

[16] Q. Du and X. Zhang, "Statistical QoS provisionings for wireless unicast/multicast of multi-layer video streams," IEEE Journal on Selected Areas in Communications, vol. 28, no. 3, pp. 420-433, 2010.

[17] H. Meshgi, D. Zhao, and R. Zheng, "Joint channel and power allocation in underlay multicast device-to-device communications," in Proceedings of the IEEE International Conference on Communications (ICC '15), pp. 2937-2942, London, UK, June 2015.

[18] X. Wu, Y. Chen, X. Yuan, and M. E. Mkiramweni, "Joint resource allocation and power control for cellular and device-to-device multicast based on cognitive radio," IET Communications, vol. 8, no. 16, pp. 2805-2813, 2014.

[19] B. Peng, C. Hu, T. Peng, Y. Yang, and W. Wang, "A resource allocation scheme for D2D multicast with QoS protection in OFDMA-based systems," in Proceedings of the IEEE 24th Annual International Symposium on Personal, Indoor, and Mobile Radio Communications (PIMRC '13), pp. 2383-2387, London, UK, September 2013.

[20] A. Bhardwaj and S. Agnihotri, "A resource allocation scheme for device-to-device multicast in cellular networks," in Proceedings of the 26th IEEE Annual International Symposium on Personal, Indoor, and Mobile Radio Communications (PIMRC '15), pp. 1498-1502, Hong Kong, China, September 2015.

[21] C. M. Huang, C. H. Lee, and H. Y. Lai, "Energy-aware group LBS using D2D offloading and M2M-based mobile proxy handoff mechanisms over the mobile converged networks," IEEE Transactions on Emerging Topics in Computing, vol. 4, no. 4, pp. 528-540, 2016.
[22] Y. Li, T. Wu, P. Hui, D. Jin, and S. Chen, "Social-aware D2D communications: qualitative insights and quantitative analysis," IEEE Communications Magazine, vol. 52, no. 6, pp. 150-158, 2014.

[23] Y. Zhao, Y. Li, Y. Cao, T. Jiang, and N. Ge, "Social-aware resource allocation for device-to-device communications underlaying cellular networks," IEEE Transactions on Wireless Communications, vol. 14, no. 12, pp. 6621-6634, 2015.

[24] F. Wang, Y. Li, Z. Wang, and Z. Yang, "Social-community-aware resource allocation for D2D communications underlaying cellular networks," IEEE Transactions on Vehicular Technology, vol. 65, no. 5, pp. 3628-3640, 2016.

[25] Y. Zhang, E. Pan, L. Song, W. Saad, Z. Dawy, and Z. Han, "Social network aware device-to-device communication in wireless networks," IEEE Transactions on Wireless Communications, vol. 14, no. 1, pp. 177-190, 2015.

[26] Y. Cao, T. Jiang, X. Chen, and J. Zhang, "Social-aware video multicast based on device-to-device communications," IEEE Transactions on Mobile Computing, vol. 15, no. 6, pp. 1528-1539, 2016.

[27] A. Orsino, L. Militano, G. Araniti, and A. Iera, "Social-aware content delivery with D2D communications support for emergency scenarios in 5G systems," in Proceedings of the 22nd European Wireless Conference (EW '16), pp. 344-349, CWC, Oulu, Finland, May 2016.

[28] G. Zhang, K. Yang, and H. Chen, "Socially aware cluster formation and radio resource allocation in D2D networks," IEEE Wireless Communications, vol. 23, no. 4, pp. 68-73, 2016.

[29] M. Zhang, X. Chen, and J. Zhang, "Social-aware relay selection for cooperative networking: an optimal stopping approach," in Proceedings of the 1st IEEE International Conference on Communications (ICC '14), pp. 2257-2262, Sydney, Australia, June 2014.

[30] Y. Cai, D. Wu, and W. Yang, "Social-aware content downloading mode selection for D2D communications," in Proceedings of the IEEE International Conference on Communications (ICC '15), pp. 2931-2936, June 2015.

[31] U. von Luxburg, "A tutorial on spectral clustering," Statistics and Computing, vol. 17, no. 4, pp. 395-416, 2007.

[32] L. Lei, Z. Zhong, C. Lin, and X. Shen, "Operator controlled device-to-device communications in LTE-advanced networks," IEEE Wireless Communications, vol. 19, no. 3, pp. 96-104, 2012.

[33] N. Zhao, F. R. Yu, and H. Sun, "Adaptive energy-efficient power allocation in green interference-alignment-based wireless networks," IEEE Transactions on Vehicular Technology, vol. 64, no. 9, pp. 4268-4281, 2015.

[34] N. Zhao, F. R. Yu, H. Sun, and M. Li, "Adaptive power allocation schemes for spectrum sharing in interference-alignment-based cognitive radio networks," IEEE Transactions on Vehicular Technology, vol. 65, no. 5, pp. 3700-3714, 2016.

[35] Y. Jiang, Q. Liu, F. Zheng, X. Gao, and X. You, "Energyefficient joint resource allocation and power control for D2D communications," IEEE Transactions on Vehicular Technology, vol. 65, no. 8, pp. 6119-6127, 2016.

[36] D. Feng, G. Yu, C. Xiong et al., "Mode switching for energyefficient device-to-device communications in cellular networks," IEEE Transactions on Wireless Communications, vol. 14, no. 12, pp. 6993-7003, 2015.

[37] D. Feng, L. Lu, Y.-W. Yi, G. Y. Li, G. Feng, and S. Li, "Device-todevice communications underlaying cellular networks," IEEE Transactions on Communications, vol. 61, no. 8, pp. 3541-3551, 2013. 
[38] H. Wu, Z. Zhong, and L. Hanzo, "A cluster-head selection and update algorithm for ad hoc networks," in Proceedings of the 53rd IEEE Global Communications Conference (GLOBECOM '10), pp. 1-5, December 2010.

[39] M. N. Soorki, M. Yaghini, M. H. Manshaei, W. Saad, and H. Saidi, "Energy-aware optimization and mechanism design for cellular device-to-device local area networks," in Proceedings of the Annual Conference on Information Science and Systems (CISS '16), pp. 309-314, Princeton, NJ, USA, March 2016.

[40] L. Al-Kanj, H. V. Poor, and Z. Dawy, "Optimal cellular offloading via device-to-device communication networks with fairness constraints," IEEE Transactions on Wireless Communications, vol. 13, no. 8, pp. 4628-4643, 2014.

[41] Q. Du, H. Song, Q. Xu, P. Ren, and L. Sun, "Interferencecontrolled D2D routing aided by knowledge extraction at cellular infrastructure towards ubiquitous CPS," Personal and Ubiquitous Computing, vol. 19, no. 7, pp. 1033-1043, 2015.

[42] L. Zhou and C. Li, "Outsourcing eigen-decomposition and singular value decomposition of large matrix to a public cloud," IEEE Access, vol. 4, pp. 869-879, 2016.

[43] E. Bastug, M. Bennis, and M. Debbah, "Living on the edge: the role of proactive caching in $5 \mathrm{G}$ wireless networks," IEEE Communications Magazine, vol. 52, no. 8, pp. 82-89, 2014.

[44] B. Peng, T. Peng, Z. Liu, Y. Yang, and C. Hu, "Cluster-based multicast transmission for device-to-device (D2D) communication," in Proceedings of the IEEE 78th Vehicular Technology Conference (VTC Fall '13), pp. 1-5, Las Vegas, Nev, USA, September 2013. 

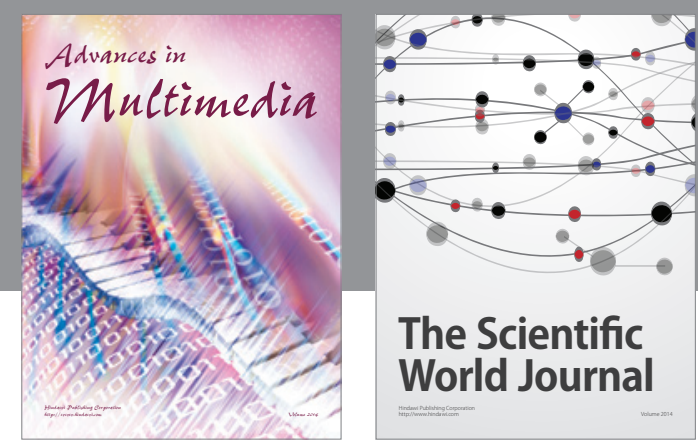

The Scientific World Journal
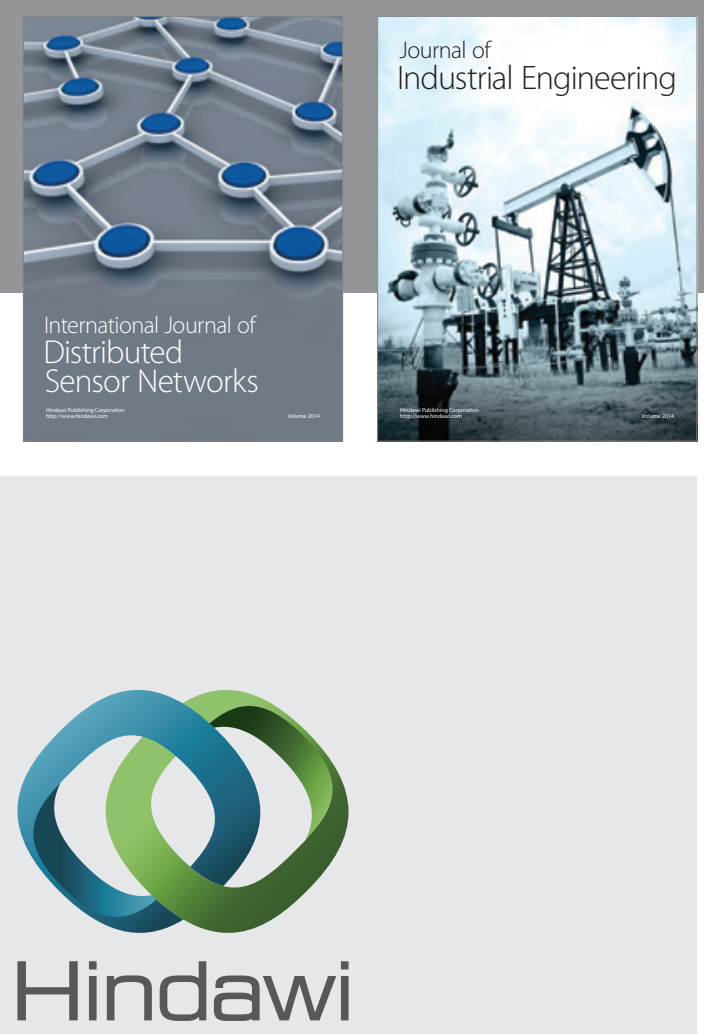

Submit your manuscripts at

https://www.hindawi.com

\section{Computer Networks} and Communications
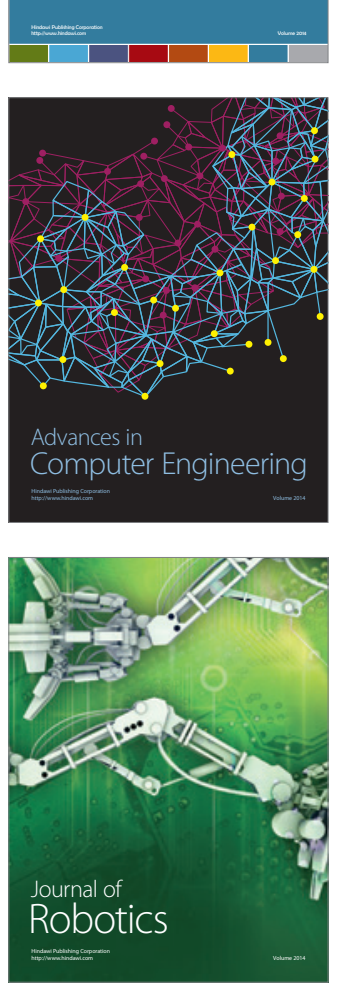
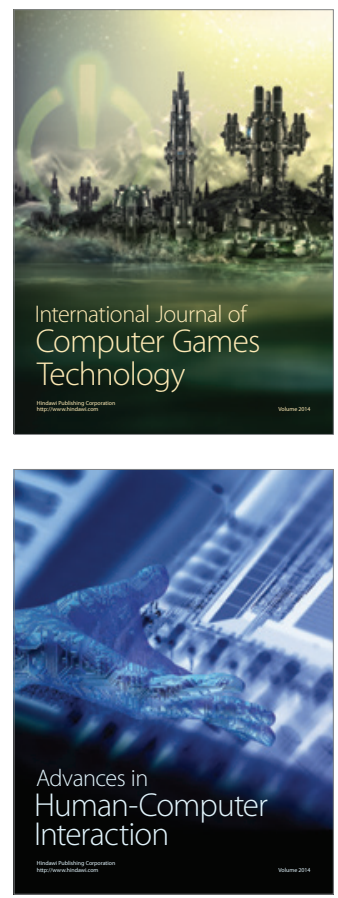
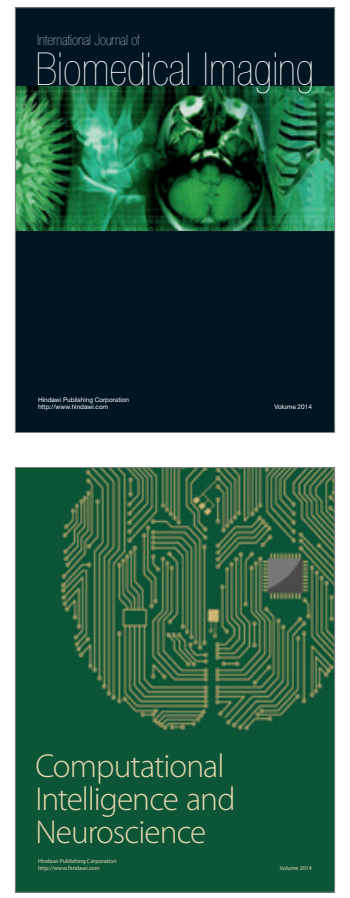
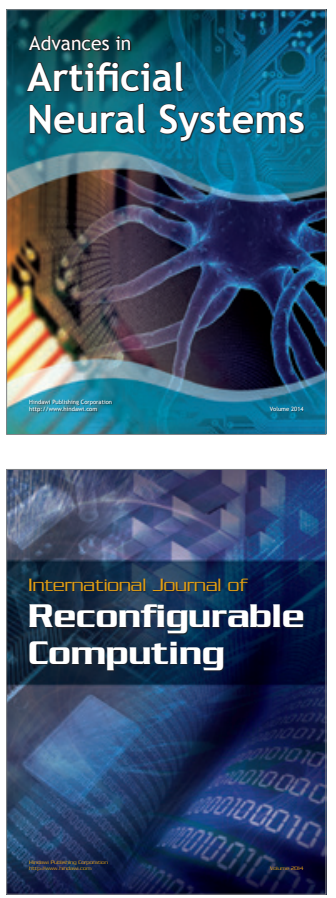
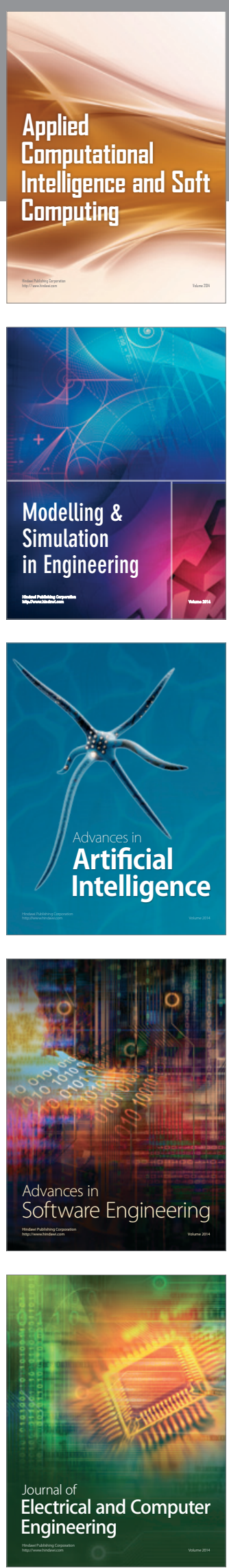